\title{
GEOMETRICAL MODELS FOR SUBSTITUTIONS
}

\author{
PIERRE ARNOUX, JULIEN BERNAT, AND XAVIER BRESSAUD
}

\begin{abstract}
We consider a substitution associated with the Arnoux-Yoccoz Interval Exchange Transformation (IET) related to the Tribonacci substitution. We construct the so-called stepped lines associated with the fixed points of the substitution in the Abelianization (symbolic) space. We analyse various projections of the stepped line recovering the Rauzy Fractal, a Peano Curve related to the work of Arnoux [1], another Peano curve related to the work of McMullen and Lowenstein, Poggiaspalla, Vivaldi [13], and also the interval exchange itself.
\end{abstract}

\section{INTRODUCTION}

1.1. Historical background. After the classification by Thurston of the automorphisms of surfaces and the definition of pseudo-automorphisms of surfaces, the question was asked to determine the possible dilation coefficients of such automorphisms; at the time, the only known examples were lifts of toral automorphisms, with quadratic coefficients.

One of the earlier non-trivial examples was a cubic one, [2], whose dilation coefficient was the real root $\lambda$ of the Tribonacci polynomial $X^{3}-X^{2}-X-1$; it was found as an application of Veech's method, by exhibiting a particular interval exchange on the unit circle which was conjugate to its first return map on an interval of length $\alpha=1 / \lambda$ (see Subsection 3.1 for details).

Around the same time, Rauzy [17] showed how to give geometric models of a particular type of substitution dynamical system as a rotation on the torus, by projecting the stepped line corresponding to the fixed point of the substitution. The first example, concerning the Tribonacci substitution on 3 letters $a \mapsto a b, \quad b \mapsto a c, \quad c \mapsto a$, also involved the Tribonacci polynomial (the characteristic polynomial of the Abelianization matrix); the two systems were soon showed to be measurably conjugate, and even semi conjugate by a continuous, onto, measure preserving map from the circle to the torus, (a Peano curve) [1]. Indeed, the interval exchange, because of the property of self induction, was easily seen as measurably conjugate to the symbolic system defined by the substitution. This example was a special case of a property, proved by Fathi [9], of semi-conjugacy of a pseudo-Anosov map to the toral automorphism defined by the homology in the hyperbolic case, where the homology matrix has no eigenvalue of modulus one.

This example was also considered by Sirvent [18, 19] and Messaoudi [15], who considered various properties. More recently, McMullen built from this example, by lifting the orbit of 0 to $\mathbb{Q}(\lambda)$ seen as $\mathbb{Q}^{3}$ a curious stepped curve with self-similarity properties (a "deterministic brownian motion") whose vertices completely filled a slice of a 3-dimensional lattice contained between two parallel planes, see [14]; this curve was studied in details by Lowenstein, Poggiapalla, Vivaldi in [13].

However, the method involved are $a d$ hoc, and difficult to generalize; they leave a number of questions opened: the partition used for the coding of the interval exchange map is only generating in measure, almost everywhere, not everywhere; the reciprocal of the 
eigenvalues, which should always be present for a self-induced interval exchange (because of the preservation of the symplectic form on the homology by the related pseudo-Anosov map) disappear at the symbolic level; and there is at the moment no systematic way to reconstruct the interval exchange from the substitution (in particular, the projection method seems to produce only group translation factors, not interval exchanges). There is also a periodic behavior of order 3 linked to the permutation of the separatrices of the singular point by the pseudo-Anosov map, which is hidden in several constructions, as in the paper [13] which considers self-similarity by the cube of the Tribonacci number.

In this paper, we present a complete study of this example, recovering all these properties in a more systematic way, and giving some insight on the structure ot the interval exchange. The basic tool is a partition in 9 intervals which is finer than the partition by continuity intervals, hence generating, and adapted to the substitution. The linear map associated with the substitution admits as characteristic polynomial the product of the Tribonacci polynomial, its reciprocal polynomial, and the polynomial $X^{3}-1$. With this substitution are associated some stepped lines in $\mathbb{R}^{9}$, coming from infinite periodic points for the substitution; this gives a unified setting from which we can obtain all the geometric objects described above (Interval exchange, Rauzy fractal, McMullen curve). By projecting these stepped lines to the various invariant subspaces, and using renormalization when needed, we recover the geometric models for this substitution as toral translation or interval exchange map, and the McMullen curve. We get a much better understanding of this dynamical system, which should be seen as defined, not on a circle, but on the disjoint union of three intervals.

This study is an example of application of some general properties of substitutions, which allows to recover all classical properties in a systematic way, and find several new ones. It raises a number of open questions.

Remark 1. This example has been much studied recently from a different viewpoint. As we explained above, the suspension of the interval exchange gives a measured foliation on a surface of genus 3, which is the stable foliation of a pseudo-Anosov map; the unstable measured foliation of this map is well defined, up to a constant, and this defines a quadratic differential on the surface (up to the action of the geodesic Teichmüller flow). This quadratic differential defines uniquely a periodic orbit for the Teichmüller flow on the modular space of quadratic differential; it also defines a Teichmüller disk for the natural action of $S L(2, \mathbb{R})$. The stabilizer of the initial quadratic differential, the so-called "Veech group" is the subject of four recent papers. It is obviously nontrivial, since it contains the derivative of the pseudo-Anosov map. In [11], Hubert and Lanneau prove that this Veech group does not contain any parabolic element; in [12], Hubert, Lanneau and Möller complete the analysis: they prove that the Veech group is not cyclic, since it contains another hyperbolic element corresponding to a different stable foliation, and the Teichmüller disc is dense in the largest possible subspace (the hyperelliptic locus of the connected component of the stratum that contains it), a behavior which is very different from the quadratic case. In [7], Bowman gives a new description of the quadratic differential in terms of Delaunay polygons; and in [16], Nipper shows that this quadratic differential does not fulfill Veech's dichotomy: there is an uncountable set of minimal but not uniquely ergodic directions.

1.2. Outline of the paper. We study the properties of several objects related to the interval exchange transformation defined in [2], using two general constructions for substitutions. 
Section 2 is basic for the rest of the paper, and gives these constructions. We define stepped lines associated with words and substitutions; for a given substitution $\sigma$ with Abelianization matrix $M_{\sigma}$, we show that the projection to the contracting space of $M_{\sigma}$ of a canonical stepped line associated with $\sigma$ is a bounded set; its closure, called the Rauzy fractal, is a union of a finite number of pieces indexed by the alphabet, with the action of a pseudo-group of translation defined on each piece (it is a map if the pieces are disjoint). If instead we project to the expanding space the finite curves corresponding to $\sigma^{n}(a)$, we obtain an unbounded sequence of sets, which can be renormalized, to converge towards a self-similar curve. The Rauzy fractal and the renormalized curve are both solutions of two graph-directed iterated function systems which are dual in a precise sense.

In Section 3 we define the basic objects of this paper: the interval exchange map $T$, a partition for the domain of $T$ (this partition is in 9 intervals, and is the base of all results of this paper), and a related substitution $\sigma$ on 9 letters, whose characteristic polynomial is the product of the Tribonacci polynomial, its reciprocal polynomial, and the polynomial $X^{3}-1$. This substitution has several factors, including, in a very natural way, the Tribonacci substitution. The Abelianization matrix of the substitution can split in a dynamical way, with a stable space $E_{s}$, an unstable space $E_{u}$, and a neutral space $E_{n}$, corresponding to the eigenvalues of modulus smaller, larger, or equal to 1 . It can also split in an arithmetic way, in the Tribonacci space $E_{T}$, the "anti-Tribonacci" space $E_{\bar{T}}$ and the neutral space, corresponding to the three factors of the characteristic polynomial on the integers. Note that $E_{T}, E_{\bar{T}}$ and $E_{n}$ are rational spaces, for which we can take as basis vectors with integral coordinates, while $E_{s}$ and $E_{u}$ are not. $E_{n}$ itself splits into the direct sum of $E_{I}$, the line associated with eigenvalue 1 , and $E_{j}$, the plane associated with eigenvalues $j$ and $\bar{j}$. When we project on one of these subspaces, it should be understood that, unless otherwise stated, we project along the supplementary space.

Our main object of study will be the stepped line associated with a periodic point of the substitution by taking the Abelianization of the prefixes. We will apply the results of Section 2 to the substitution defined in Section 3, and obtain several objects which give new light, in particular, to the nature of this interval exchange, which should not be considered as defined on a circle, but on three disjoint intervals, which have a natural interpretation on the surface obtained as a suspension of the interval exchange.

In Section 4, we shall project on the space $E_{T, s}=E_{T} \cap E_{s}$, and recover the Rauzy fractal in a natural way. By projecting on $E_{\bar{T}, s} \oplus E_{j}$, we obtain three intervals; by projecting these three intervals in an appropriate way (not along $E_{j}$ ), we recover the initial interval exchange. We can also project on the complete stable space $E_{s}$; we obtain a bounded set. If we choose correctly an appropriate direction of projection, the image turns out to be a continuous curve in $\mathbb{R}^{3}$, of Hausdorff dimension 2 . This curve projects in a one-to-one way on $E_{\bar{T}, s}$ (it is strictly increasing in this direction), and it also projects one-to-one, except a set of measure 0 , on the plane $E_{T, s}$; it is in fact the graph of the Peano curve given by the semi-conjugacy of the interval exchange to a torus rotation, which appears naturally by projection.

In Section 5, we project to $E_{n}$, and prove that the projection of the stepped line is a discrete set, whose projection to $E_{I}$ is unbounded, but whose projection on $E_{j}$ is finite, consisting of three points; these three points correspond to a partition in three intervals of the domain of the interval exchange. They provide a more conceptual way to recover the results of Section 4 . 
In Section 6, we consider the projection to the unstable space, and obtain by renormalization a set of finite curves of high Hausdorff dimension. We will also consider the projection to the space $E_{\bar{T}}$. We obtain a discrete set contained in a slice of $\mathbb{Z}^{3}$; the projection of this set to a plane is an approximation of McMullen's curve, which has the same renormalization. If we use also the space $E_{j}$, we can obtain exactly McMullen's curve as a projection of the stepped line. We can in this way recover the results of Lowenstein et al. [13] in a systematic way.

In Section 7, we use the order structure given by the interval exchange map to define a new substitution dual to the first one, for which we can also study fixed points and the projections to various spaces. Remark that the definition of the dual substitution is not trivial; the matrix of this dual substitution is uniquely defined as the transpose of the matrix of the initial substitution. But this matrix is not sufficient to define a substitution: it only defines the Abelianization of the images of letters; to recover the ordering of the words, we need to use the order structure on the circle, and the hierarchy of tilings given by the induction. In the general case, when there is no natural order structure on the underlying space, the definition of a dual substitution is unclear. In the present case, it appears that the renormalized curves (projections of the stepped lines on the unstable spaces) converge to the fractals defined in Section 4. This is not completely surprising: changing the substitution to its dual is a first transposition, and changing from stable to unstable space entails a second transposition, which gives back the initial situation.

In Section 8, we state some remarks and open questions; in particular, we briefly discuss a flat structure on a surface of genus 3 associated with the interval exchange map; complete consideration of this surface would have lengthened too much this paper, but it underlies a large part of this work.

In the appendix (Section 9) we give the proof of some computational results used in the paper.

\section{Substitutions And GeOMetrical MOdels}

2.1. Substitution dynamical systems and coding. Given a finite set $\mathcal{A}$ (the alphabet), a substitution on the alphabet $\mathcal{A}$ is a non-erasing morphism on the free monoid $\mathcal{A}^{*}$; it is completely defined by a map $\mathcal{A} \rightarrow \mathcal{A}^{*}$ which sends each letter to a nonempty word. Such a substitution $\sigma$ is primitive if there exists $n$ such that, for all letter $i$, the word $\sigma^{n}(i)$ contains all letters of the alphabet. Primitive substitutions have a finite number of periodic points which are infinite sequences; the closure of the orbit under the usual shift map of any of these periodic points defines the symbolic dynamical system $\left(\Omega_{\sigma}, S\right)$ associated with the substitution. In this paper, we will only deal with primitive substitutions.

For a dynamical system $(X, T)$ and a finite partition of $X, \mathcal{I}=\left\{I_{i} \mid i \in \mathcal{A}\right\}$, we define the coding function $\nu_{\mathcal{I}}: X \rightarrow \mathcal{A}$ by $\nu_{\mathcal{I}}(x)=i$ if $x \in I_{i}$, and we define the itinerary of a point $x \in X$ under $T$ with respect to the partition $\mathcal{I}$ as the sequence $\left(\nu_{\mathcal{I}}\left(T^{n} x\right)\right)_{n \in \mathbb{N}}$. We will denote by $\varphi_{\mathcal{I}}$ the map that send a point to its itinerary.

If $A$ is a subset of $X$, and $x \in A$, we define the first-return time of $x$ as $n_{x}=\inf \{n \in$ $\left.\mathbb{N}^{*} \mid T^{n}(x) \in A\right\}$ (it is infinite if the orbit of $x$ does not come back to $A$; this will not occur in the cases we consider). If the first-return time is finite for all $x \in A$, we define the induced map of $T$ on $A$ (or first-return map) as the map $x \mapsto T^{n_{x}}(x)$, and we denote this map by $T_{A}$. 
Substitutions can be used to define symbolic dynamics for self-induced dynamical systems; more precisely:

Definition 2. Let $(X, T)$ be a dynamical system, let $\sigma$ be a substitution on the alphabet $\mathcal{A}$, and let $\mathcal{I}=\left\{I_{i} \mid i \in \mathcal{A}\right\}$ be a partition of $X$ indexed by $\mathcal{A}$. We say that the dynamical system has $\sigma$-structure with respect to the partition $\mathcal{I}$ if there exists a subset $A \subset X$, and a bijection $h: X \rightarrow A$, such that:

(1) The map $h$ conjugate $T$ to $T_{A}: T=h^{-1} \circ T_{A} \circ h$.

(2) The first-return time is constant, denoted by $n_{i}$, on each set $J_{i}=h\left(I_{i}\right)$.

(3) The sets $T^{k}\left(J_{i}\right)$, for $i \in \mathcal{A}$ and $0 \leq k<n_{i}$, form a partition which refines the partition $\mathcal{I}$.

(4) For $x \in J_{i}$, the finite coding $\left(\nu_{\mathcal{I}}\left(T^{k} x\right)\right)_{0 \leq k<n_{i}}$ with respect to the partition $\mathcal{I}$ is given by the word $\sigma(i)$.

If a system has $\sigma$-structure with respect to a partition, it is semi-conjugate (by the itinerary map $\varphi_{\mathcal{I}}$ ) to the symbolic dynamical system associated with $\sigma$; if the sequence of partitions obtained by iterating the map $h$ is generating (for example if the diameter of the elements of this sequence of partition tends to 0 ), this semi-conjugacy $\varphi_{\mathcal{I}}$ is in fact one-to-one. In this paper, we will explore some examples of dynamical systems with $\sigma$-structure for a substitution.

To a substitution $\sigma$, we can associate the occurence matrix $M_{\sigma}=\left(m_{i, j}\right)_{i, j \in \mathcal{A}}$ indexed by $\mathcal{A}$, where $m_{i, j}$ is the number of occurences of $i$ in $\sigma(j)$. A substitution $\sigma$ is primitive if and only if $M_{\sigma}$ is primitive in the usual sense of matrices. In that case, the classical Perron-Frobenius theorem applies, and the matrix has a dominant eigenvalue, and an associated positive eigenvector.

2.2. Prefix automaton. Let $u$ be an infinite periodic point for the substitution $\sigma$, and let $u_{1} \ldots u_{n}$ be the prefix of length $n$ of $u$. Since we can find $v$ such that $u=\sigma(v)$, we can write $u_{1} \ldots u_{n}=\sigma(V) P_{0}$, where $P_{0}$ is a strict suffix of $\sigma\left(a_{0}\right)$ and $V a_{0}$ is a prefix of $v$; we can iterate the process, and find a sequence $P_{0}, P_{1}, \ldots, P_{k}$ of prefixes of images of letters such that $u_{1} \ldots u_{n}=\sigma^{k}\left(P_{k}\right) \sigma^{k-1}\left(P_{k-1}\right) \ldots \sigma\left(P_{1}\right) P_{0}$.

This sequence of prefix is not arbitrary; it satisfies a combinatorial condition given by the prefix automaton.

Definition 3. The prefix automaton associated with a substitution $\sigma$ is the automaton whose states are indexed by the letters of the alphabet $\mathcal{A}$, and whose transitions are labeled by the strict prefixes of images of letters. There is a transition from state a to state $b$, indexed by $P$, if $P a$ is a prefix of $\sigma(b)$.

Example 4. The Tribonacci substitution $\breve{\sigma}$ is defined on the alphabet $\{1,2,3\}$ by $\breve{\sigma}(1)=$ $12, \breve{\sigma}(2)=13, \breve{\sigma}(3)=1$. The only nonempty prefix is 1 ; the prefix automaton is shown below, and the admissible sequences of prefixes are exactly those which do not contain three consecutive 1. 


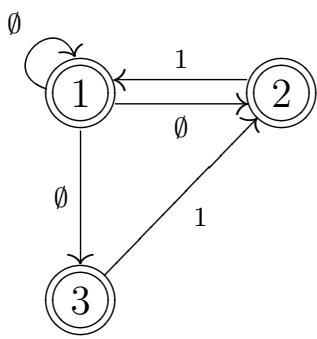

\subsection{Canonical stepped line associated with a substitution.}

\subsubsection{Stepped lines: basic definitions.}

Definition 5. A stepped line $\boldsymbol{L}=\left(x_{n}\right)_{n \geq 0}$ in $\mathbb{R}^{d}$ is a sequence (it could be finite or infinite) of points in $\mathbb{R}^{d}$ such that the steps $x_{n+1}-x_{n}$ belong to a finite set.

$A$ canonical stepped line $i s$ a stepped line such that $x_{0}=0$ and for all $n \geq 0, x_{n+1}-x_{n}$ belongs to the canonical basis $\mathcal{B}$ of $\mathbb{R}^{d}$.

If $\mathcal{A}$ is a finite alphabet, there is an obvious bijection between canonical stepped lines in $\mathbb{R}^{\mathcal{A}}$ and words on the alphabet $\mathcal{A}$, which we will make explicit:

Definition 6. The Abelianization map $e: \mathcal{A}^{*} \rightarrow \mathbb{Z}^{\mathcal{A}}$, is defined for any $W \in \mathcal{A}^{*}$ by $e(W)=\left(|W|_{a}\right)_{a \in \mathcal{A}}$, where $|W|_{a}$ is the number of occurences of the letter a in $W$.

It will be convenient to use the notation $e_{a}=e(a)$ for the basis vector, and to write, for any word $W=w_{1} \ldots w_{n}$ of length $n$ in $\mathcal{A}^{*}, e_{W}=e(W)=\sum_{k=1}^{n} e_{w_{k}}$.

Using this map, to any finite or infinite word $W$, we can associate a canonical stepped line $\mathbf{L}^{W}$ in $\mathbb{R}^{\mathcal{A}}$ as the sequence $\left(e_{P_{n}}\right)$, where the $P_{n}$ are the prefixes of length $n$ of $W$. We will sometimes refer to the (non ordered) set $\mathcal{V}^{W}=\cup_{n \geq 0}\left\{x_{n}^{W}\right\}$ of vertices of the stepped line. We will denote $\mathbf{L}_{n}^{W}$ (resp. $\mathcal{V}_{n}^{W}$ ) the finite stepped line associated with the prefix of $W$ of length $n$ (resp. its set of vertices).

Remark 7. A canonical stepped line is a discrete set of point. There is a natural geometric curve underlying this stepped line, made of oriented segments linking the successive vertices of the stepped line (we let the reader supply the formal definition). This underlying curve will appear in Subsection 2.5 and in Section 6.

2.3.2. Stepped lines associated with substitutions: combinatorial properties. We now fix a substitution $\sigma$. This paper will be concerned with a particular type of canonical stepped line:

Definition 8. An infinite canonical stepped line associated with a periodic point of the substitution $\sigma$ is called a canonical stepped line associated with $\sigma$.

Recall that, by definition, the Abelianization map satisfies the relation, for any word $W \in \mathcal{A}^{*}:$

$$
e_{\sigma(W)}=M_{\sigma} e_{W}
$$

From this, we deduce easily a first decomposition of the prefixes of the canonical stepped lines associated with $\sigma$. 
Proposition 9. For any letter $a \in \mathcal{A}$, we have a disjoint decomposition:

$$
\mathcal{V}^{\sigma^{n+1}(a)}=\coprod_{P, b \mid P b \text { prefix of } \sigma(a)} \mathcal{V}^{\sigma^{n}(b)}+e_{\sigma^{n}(P)}=\coprod_{P, b ; P b \text { prefix of } \sigma(a)} \mathcal{V}^{\sigma^{n}(b)}+M_{\sigma}^{n} e_{P}
$$

Proof. This is a direct consequence of the trivial relation $\sigma^{n+1}(a)=\sigma^{n}(\sigma(a))$.

This decomposition is only valid for finite stepped lines; a slightly more subtle one, valid for infinite stepped lines, is related to the following notion:

Definition 10. Let $u \in \mathcal{A}^{\mathbb{N}}$. The point $x_{n} \in \boldsymbol{L}^{u}$ is of type a if $u_{n+1}=a$. We denote by $\mathcal{V}^{u, a}$ the sets of vertices of type a of $\boldsymbol{L}^{u}$.

The fixed points of a substitution satisfy the following similarity property:

Proposition 11. Let $u$ be an infinite fixed point of the substitution $\sigma$. Then we have the disjoint decomposition:

$$
\mathcal{V}^{u, a}=\coprod_{P, b \mid \text { Pa prefix of } \sigma(b)} M_{\sigma} \mathcal{V}^{u, b}+e_{P}
$$

Proof. This is a simple consequence of the fact that, if $x_{n}$ is of type $a$, then the corresponding prefix $P_{n+1}=P_{n} a$ of the fixed point $u$ can be written $P_{n+1}=\sigma\left(P_{k}\right) P a$, where $P_{k+1}=P_{k} b$ and $P a$ is a prefix of $\sigma(b)$.

Remark 12. These relations can be seen as examples of graph-directed iterated function systems, except for the fact that the maps involved are not contracting. Note that the graph involved in proposition 9 is the opposite of the graph involved in proposition 11 (the direction of the edges of the graph are reversed), and the associated adjacency matrix is transposed; these two relations are, in some sense, dual.

2.4. Stepped lines in contracting spaces and Rauzy fractals. A basic property of a canonical stepped line associated with a substitution is the following:

Proposition 13. Let $E \oplus F$ be a splitting of $\mathbb{R}^{\mathcal{A}}$ which is invariant under the action of $M_{\sigma}$. If the restriction of $M_{\sigma}$ to $E$ is strictly contracting, then any canonical stepped line associated with $\sigma$ stays within bounded distance of $F$.

Proof. Using the prefix automaton, we can decompose any prefix of a periodic point as $\sigma^{k}\left(P_{k}\right) \sigma^{k-1}\left(P_{k-1}\right) \ldots \sigma\left(P_{1}\right) P_{0}$. The image of this prefix by the Abelianization map is $e\left(\sigma^{k}\left(P_{k}\right) \sigma^{k-1}\left(P_{k-1}\right) \ldots \sigma\left(P_{1}\right) P_{0}\right)=\sum_{i=0}^{k} e\left(\sigma^{i}\left(P_{i}\right)\right)=\sum_{i=0}^{k} M_{\sigma}^{i} . e_{P_{i}}$. The projection of this sum on $E$ is a sum whose general term decreases geometrically fast, hence this projection is bounded.

This proposition has an immediate consequence:

Corollary 14. Let $E \oplus F$ be a splitting of $\mathbb{R}^{\mathcal{A}}$ which is invariant under the action of $M_{\sigma}$. If the restriction of $M_{\sigma}$ to $E$ is strictly contracting, the projection of the vertices of the canonical line on $E$ along $F$ is a bounded set. 
In case of the projection on the contracting space associated with a substitution (and specially in the case of a substitution of Pisot type), the closure of this set is what is usually called the Rauzy fractal of the substitution, denoted by $\mathcal{R}$. This Rauzy fractal has remarkable properties; first of all, it is the solution of a graph-directed iterated function system (GIFS for short).

Proposition 15. Let $\mathcal{R}_{a}$ be the closure of the projection of the vertices of type a, for all $a \in \mathcal{A}$. The sets $\mathcal{R}_{a}$ satisfy the relation:

$$
\mathcal{R}_{a}=\coprod_{P, b \mid \text { Pa prefix of } \sigma(b)} M_{\sigma} \mathcal{R}_{b}+e_{P}
$$

This is an immediate consequence of the proposition 11; note that, since the restriction of $M_{\sigma}$ to $E$ is a contraction, this relation uniquely defines the $\mathcal{R}_{a}$ and their union $\mathcal{R}$.

We can define an additional dynamical structure on $\mathcal{R}$. There is a natural order on the vertices of a stepped line; it allows us to define a map on the set of vertices, which takes any vertex to the successor. If a vertex $P$ is of type $a$, the next vertex is by definition $P+e_{a}$. By projection, the successor function is well-defined on a dense subset of $\mathcal{R}_{a}$; it is a translation by the projection of $e_{a}$, hence continuous, so it extends by continuity to all of $\mathcal{R}_{a}$. This defines, in the general case, a pseudo-group of transformation acting on the subsets $\mathcal{R}_{a} \subset \mathcal{R}$. If these sets are disjoint, each point is in the domain of exactly one generator of the pseudogroup, and the successor map extends to all of $\mathcal{R}$ (except maybe on boundaries of $\mathcal{R}_{a}$ ), defining an exchange of pieces (in a number of interesting cases, the set are disjoint up to a set of measure 0, and the map is defined except on a set of measure 0).

Example 16 (The original Rauzy fractal). A classical example, which is the basis for this paper, is the Tribonacci substitution $\breve{\sigma}$ first studied by Rauzy and defined above, in Example 4. The associated matrix has an unstable space of dimension 1 (the PerronFrobenius expanding line) and a stable space of dimension 2, on which the action of the Abelianized matrix is conjugate to the action on $\mathbb{C}$ by multiplication by $\mu$, where $\mu$ is one of the two complex contracting eigenvalues.

Using the prefix automaton, and choosing a complex structure on the contracting plane such that $f(1)$ projects to 1 and the action of the Abelianized matrix corresponds to multiplication by $\mu$, we see that any vertex of the stepped line associated with the substitution projects on the contracting plane to a finite sum $\sum_{0}^{k} \epsilon_{i} \mu^{i}$, where $\left(\epsilon_{i}\right)$ is a sequence of 0 and 1 such there are no three consecutive 1. The closure of the projection on the contracting plane is the Rauzy fractal (as defined above) of the Tribonacci substitution; we will call it the Tribonacci fractal for short, denoted by $\breve{\mathcal{R}}$.

2.5. Stepped lines in expanding spaces and fractal curves. Instead of projecting on a contracting space, we can project on an expanding space; in that case, we obtain a discrete line that tends to infinity. We will show that, by renormalizing in a suitable way this discrete line, we can obtain another interesting object.

2.5.1. Projection on Perron-Frobenius eigenline, tiling and number system. Let $\lambda$ be the Perron-Frobenius eigenvalue, and let $E_{\lambda} \oplus E_{\lambda}^{*}$ the invariant splitting given by the PerronFrobenius eigenline and its invariant complement. We denote $\Pi_{\lambda}$ the projection on $E_{\lambda}$ along $E_{\lambda}^{*}$; its restriction to the geometric curve underlying any canonical stepped line is 
a bijection, since $E_{\lambda}^{*}$ is the orthogonal of the dual Perron-Frobenius eigenvector, which is strictly positive, hence the curve intersects exactly once each hyperplane parallel to $E_{\lambda}^{*}$.

This projection gives us a natural tiling of $E_{\lambda}$, which is self-similar. Is also gives us a parametrization of the canonical line, and a number system, in the following way.

Let $a \in \mathcal{A}$. The stepped line $\mathbf{L}^{\sigma^{n}(a)}$ projects to the interval $\left[0, \Pi_{\lambda} e_{\sigma^{n}(a)}\right]=\left[0, \lambda^{n} \Pi_{\lambda} e_{a}\right]$ of $E_{\lambda}$. It is an immediate consequence of the definition of the prefix automaton that, for any vertex $x \in \mathcal{V}^{\sigma^{n}(a)}$, we can find a finite sequence $\left(P_{1}, \ldots, P_{n}\right)$ of prefixes, and a sequence $\left(a_{0}=a, \ldots, a_{n}\right)$ of letters, such that $P_{k} a_{k}$ is a prefix of $\sigma\left(a_{k-1}\right)$ for $k=1, \ldots, n$. The corresponding word can then be written $\sigma^{n-1}\left(P_{1}\right) \ldots \sigma^{k}\left(P_{n-k}\right) \ldots P_{n}$, and an immediate computation shows that we can write:

$$
\Pi_{\lambda}(x)=\sum_{k=1}^{n} \lambda^{n-k} \Pi_{\lambda}\left(e_{P_{k}}\right) .
$$

If we apply an homothety of ratio $\lambda^{-n}$ to $E_{\lambda}$, the interval $\left[0, \Pi_{\lambda} e_{\sigma^{n}}(a)\right]$ is sent to $\left[0, \Pi_{\lambda} e_{a}\right]$, and it is not difficult to check that, for any $y$ in this interval, there is a uniquely defined sequence $\left(P_{n}\right)_{n \in \mathbb{N}}$ which is admissible in the sense above, and such that $y=$ $\sum_{k=1}^{\infty} \lambda^{-k} \Pi_{\lambda}\left(e_{P_{k}}\right)$; it can be seen as an expansion in base $\lambda$, the digits being the $\Pi_{\lambda}\left(e_{P}\right)$, for all prefixes of images of letters; they form a finite set.

Remark 17. This construction was already considered and studied in detail by Dumont and Thomas, see [8]. There is not one number system, but a finite collection, one system for each letter. If the letter a is the beginning of an infinite word which is fixed or periodic for the substitution, this number system extends in a natural way to the positive line, but not otherwise.

Remark that the sequence of prefixes is determined by the prefix automaton, with the arrows reversed. A point $x$ in the interval has a finite expansion if and only if there is $N \in \mathbb{N}$ such that $\lambda^{N} x$ is a vertex of the tiling obtained by projecting $\sigma^{N}(a)$ (the property is then true for all $n \geq N$ ).

2.5.2. Stepped lines in expanding spaces. Let $E \oplus F$ be an invariant splitting of $\mathbb{R}^{\mathcal{A}}$, such that the restriction of $M_{\sigma}$ to $F$ is strictly expanding, and denote by $\Pi_{F}$ the projection on $F$ along $E$. We consider the projection of the stepped lines $\mathbf{L}^{\sigma^{n}(a)}$ on $F$. It is clear that, unless $\Pi_{F}\left(e_{a}\right)=0$, these stepped lines tend to infinity, since their endpoint are $\Pi_{F}\left(e_{\sigma^{n}(a)}\right)=M_{\sigma}^{n} \Pi_{F}\left(e_{a}\right)$. We will show that, after an appropriate renormalization, these stepped lines converge. We start by an elementary fact.

Lemma 18. For all $a \in \mathcal{A}$ and all $0 \leq i \leq|\sigma(a)|$,

$$
\Pi_{F}\left(x_{i}^{\sigma^{n}(a)}\right)=\sum_{k=1}^{n} M_{\sigma}^{n-k} \Pi_{F}\left(e_{P_{k}}\right),
$$

where $\sigma^{n-1}\left(P_{1}\right) \cdots \sigma\left(P_{n-1}\right) P_{n}$ is the prefix decomposition of the prefix of $\sigma^{n}(a)$ of length $i$ (remark that this decomposition is ordered in the opposite direction compared to the previous section).

Proof. The existence of the prefix decomposition of any prefix $P$ of $\sigma^{n}(a)$, given by $P=\sigma^{n-1}\left(P_{1}\right) \cdots \sigma\left(P_{n-1}\right) P_{n}$ has been shown above by the analysis of the prefix automaton. It immediately implies the formula. 
From this lemma, we easily prove:

Lemma 19. For all $a \in \mathcal{A}$, the set $\overline{\cup_{n \geq 0} M^{-n} \Pi_{F}\left(\mathcal{V}^{\sigma^{n}(a)}\right)}$ is a compact connected set.

Proof. Since $M \mathcal{V}^{\sigma^{n}(a)} \subset \mathcal{V}^{\sigma^{n+1}(a)}$, the sets $M^{-n} \Pi_{U}\left(\mathcal{V}^{\sigma^{n}(a)}\right)$ form an increasing sequence. They are uniformly bounded, by an argument similar to the argument that proves the existence of the Rauzy fractal, the geometric series being taken in the other direction. Indeed, let $y \in M^{-n} \Pi_{U}\left(\mathcal{V}^{\sigma^{n}(a)}\right)$. By the previous lemma, we can write $y=\sum_{j=1}^{n} M_{\sigma}^{-j} \Pi_{F}\left(e_{P_{j}}\right)$. Since the $e_{P}$ form a finite set, and $M_{\sigma}$ is a strict contraction on $F$, this sum is uniformly bounded by a geometric series.

A similar argument shows that this set is well-chained, hence connected.

The limit set is in fact the image of a parametrized curve, which can be given explicitly. We have shown that the projection $\Pi_{\lambda}$ is a bijection from the curve underlying the canonical stepped line $\mathbf{L}^{\sigma^{n}(a)}$ to the interval $\left[0, \lambda^{n} \Pi_{\lambda}\left(e_{a}\right)\right]$. We denote by $\gamma$ the reciprocal map (that is, the natural parametrization of the curve by the Perron-Frobenius space).

We define a sequence of parametrized curves $\mathcal{C}_{n}^{a}:\left[0, \Pi_{\lambda}\left(e_{a}\right)\right] \rightarrow F$ by

$$
\mathcal{C}_{n}^{a}(t)=M^{-n} \Pi_{F}\left(\gamma\left(\lambda^{n} t\right)\right)
$$

Proposition 20. The sequence of parametrized curves $\mathcal{C}_{n}^{a}$ tends uniformly to a curve $\mathcal{C}^{a}$, which is defined by:

$$
\mathcal{C}^{a}(t)=\sum_{j=1}^{\infty} M_{\sigma}^{-j} \Pi_{F}\left(e_{P_{j}}\right),
$$

where $t \Pi_{\lambda}\left(e_{a}\right)=\sum_{j=1}^{\infty} \lambda^{-j} \Pi_{\lambda}\left(e_{P_{j}}\right)$ is the expansion of $t \Pi_{\lambda}\left(e_{a}\right)$ in the Dumont-Thomas number system in base $\lambda$ associated with a (as in Remark 17).

Proof. This is a simple computation. If $t$ has a finite expansion of degree $N$, all $\mathcal{C}_{n}^{a}(t)$ are equal for $n>N$, and given by the formula above; otherwise, they converge geometrically fast to it.

We get in this way a family of curves $\left(\mathcal{C}^{a}\right)_{a \in \mathcal{A}}$; as we shall see later, they are quite often of the Peano type, with dimension greater than 1 . Their images $\mathcal{R}_{a}^{*}=\left\{\mathcal{C}^{a}(t) \mid 0 \leq t \leq \Pi_{\lambda}\left(e_{a}\right)\right\}$ satisfy an interesting relation:

Proposition 21. We have:

$$
\mathcal{R}_{a}^{*}=\bigcup_{P, b \mid \text { Pb prefix of } \sigma(a)} M^{-1}\left(\mathcal{R}_{b}^{*}+e_{P}\right) .
$$

Proof. The sets $M^{-n} \mathcal{V}^{\sigma^{n}(a)}$ are contained in $\mathcal{R}_{a}^{*}$, and their union is dense. The proposition is then an immediate consequence of the proposition 9 .

We observe again that we have a graph directed IFS, which is dual to the one defined for the Rauzy fractal; the Rauzy fractal $\mathcal{R}$ and its dual $\mathcal{R}^{*}$ live in different spaces, and they have no reason to be similar.

\subsection{Stepped lines associated with interval exchange transformations.}


2.6.1. Bounded stepped line in one-dimensional space. There is a completely different way to build a bounded stepped line, independently of any substitution. Consider a minimal interval exchange transformation $T$ on $k$ intervals $I_{1}, \ldots I_{k}$ of translation length $t_{1}, \ldots, t_{k}$ (that is, $T(x)=x+t_{i}$ if $x \in I_{i}$ ). Take any point $x$ in the domain, and consider the itinerary with respect to the partition; we obtain an infinite word, and a canonical stepped line in $\mathbb{R}^{k}$. Consider the projection from $\mathbb{R}^{k}$ to $\mathbb{R}$ which sends $e_{i}$ to $t_{i}$. It is clear that the image of the canonical stepped line is bounded, the closure of its image is the domain of $T$, and the exchange of pieces associated with this stepped line is well defined, and is the initial map $T$. Indeed, the image of the stepped line is, by definition, the orbit of $x$ by $T$. This stepped line can be realized in $\mathbb{R}^{k}$ by projecting on a one-dimensional space along the plane $\sum t_{i} x_{i}=0$.

If the map $T$ is self induced on a subinterval, and if we take for $x$ the fixed point of the map that conjugates $T$ to the induced map, the itinerary of $x$ is the fixed point of a substitution.

However, it is in general difficult, for a given substitution $\sigma$, to find corresponding translations lengths $t_{i}$, to check whether the substitution dynamical system is conjugate (at least measurably) to an interval exchange transformation, and to recover the interval exchange in a systematic way; we will do it below for a particular substitution.

2.6.2. Discrete stepped lines associated with algebraic interval exchanges. The following construction, due to McMullen [14], associates to any algebraic interval exchange transformation a discrete curve on a slice of a lattice.

Let $\alpha$ be an algebraic integer of degree $d$, and suppose that all the lengths of the intervals, and hence all the translation lengths, belong to $\frac{1}{N} \mathbb{Z}[\alpha]$ for some $N$. Then the orbit of 0 is in $\frac{1}{N} \mathbb{Z}[\alpha]$, which can be considered as a lattice $\mathbb{Z}^{d}$ by taking the vector $\left(n_{0}, \ldots, n_{d-1}\right) \in \mathbb{Z}^{d}$ to $\sum_{i=0}^{d-1} \frac{n_{i}}{N} \alpha^{i}$; we define in this way a stepped line in $\mathbb{Z}^{d}$, which is included in a slice of $\mathbb{Z}^{d}$ since all points satisfy $\sum_{i=0}^{d-1} \frac{n_{i}}{N} \alpha^{i} \in I$, where $I$ is the domain of the interval exchange.

All self-similar interval exchange maps, and in particular all interval exchange maps related to pseudo-Anosov maps, are algebraic, and allow this construction; we will see a particular case below.

\section{The SETting: DEFinitions AND NOTATiONS}

\subsection{The interval exchange transformation.}

3.1.1. The transformation. For any interval $[a, b) \subset[0,1)$, we define the involution $I_{[a, b)}$ on $[0,1)$ which exchanges by a translation the two halves of the interval $[a, b)$ and fixes the complement. This involution is explicitly given by

$$
\begin{aligned}
I_{[a, b)}: x & \mapsto x+\frac{b-a}{2} \quad \text { if } a \leq x<\frac{a+b}{2} \\
x & \mapsto x-\frac{b-a}{2} \text { if } \frac{a+b}{2} \leq x<b \\
x & \mapsto x \text { if } x \notin[a, b) .
\end{aligned}
$$

Let $\alpha$ be the unique real number such that $\alpha^{3}+\alpha^{2}+\alpha=1$. Define the partition $\mathcal{J}=$ $\left\{J_{a}, J_{b}, J_{c}\right\}=\left\{[0, \alpha),\left[\alpha, \alpha+\alpha^{2}\right),\left[\alpha+\alpha^{2}, 1\right)\right\}$. The map $T$ is defined as $I_{[0,1)} \circ I_{J_{a}} \circ I_{J_{b}} \circ I_{J_{c}}$ : 
we cut each of the three intervals in two equal halves, exchange these halves by translation, and then rotate the circle by $\frac{1}{2}$.

The map $T$ is an interval exchange map on the circle with 6 intervals of continuity (if we consider it as an interval exchange on the interval $[0,1)$, another discontinuity is introduced by the preimage of 0 , and we get an interval exchange map on 7 intervals). $T$ is conjugate to its inverse, by $T=I_{[0,1)} \circ T^{-1} \circ I_{[0,1)}$.

The map $T$ has $\breve{\sigma}$-structure with respect to the partition $\mathcal{J}$ for the Tribonacci substitution $\breve{\sigma}$ (see [1]). More precisely, let $h$ be the map $h:[0,1) \rightarrow J_{a}=[0, \alpha)$ defined by:

$$
\begin{aligned}
h: x & \mapsto \alpha x+\frac{\alpha+\alpha^{4}}{2} \text { if } x<\frac{\alpha+\alpha^{2}}{2} \\
x & \mapsto \alpha x+\frac{-\alpha+\alpha^{4}}{2} \text { if } x \geq \frac{\alpha+\alpha^{2}}{2} .
\end{aligned}
$$

A straightforward computation proves that $h$ conjugates $T$ to the induced map $T_{J_{a}}$; the first-return time is 1 on $h\left(J_{c}\right)$, and 2 on its complement; we can check easily that $J_{a}=h\left(J_{a}\right) \cup h\left(J_{b}\right) \cup h\left(J_{c}\right), J_{b}=T\left(h\left(J_{a}\right)\right), J_{c}=T\left(h\left(J_{b}\right)\right)$.

Hence we obtain a semi-conjugacy between the interval exchange map $T$ and the Tribonacci shift. One can prove that this semi-conjugacy is a measurable conjugacy, that is, it is almost everywhere one-to-one, but it is not one-to-one everywhere, because the partition $\mathcal{J}$ is not generating; indeed, because of the discontinuity in $h, h\left(J_{a}\right)$ is not an interval, but the union of two disjoint intervals, and $h^{2}\left(J_{a}\right)$ has three connected components, as well as the $h^{n}\left(J_{a}\right)$ for $n>2$.

We define the points $\alpha_{1}=\frac{\alpha^{4}}{2}=\alpha-\frac{1}{2}, \alpha_{2}=\alpha^{2}+\frac{\alpha^{4}}{2}=\alpha+\alpha^{2}-\frac{1}{2}$ and $\alpha_{3}=\frac{1}{2}$. They cut $\mathbb{S}^{1}$ into 3 intervals which are, respectively, $T\left(J_{a}\right), T\left(J_{b}\right)$ and $T\left(J_{c}\right)$. A computation proves that $h\left(\alpha_{1}\right)=\alpha_{2}, h\left(\alpha_{2}\right)=\alpha_{3}, h\left(\alpha_{3}\right)=\alpha_{1}$, that is, $\left\{\alpha_{1}, \alpha_{2}, \alpha_{3}\right\}$ is a periodic orbit for $h$. One can prove that $\cap_{n \in \mathbb{N}} h^{n}\left(J_{a}\right)$ consists of these three points, which have the same itinerary with respect to $\mathcal{J}$ (the fixed point of $\sigma$ ).

3.1.2. The partitions. The starting point of the present paper is the partition presented in this section; it has the property that its element are continuity intervals of the interval exchange map, and their images by the conjugacy are also intervals.

We would like to obtain a partition by intervals with a $\sigma$-structure. For this, we must introduce the discontinuity point of $h$. This in turn introduces new cutting points, and computation shows that, to obtain a $\sigma$-structure with a partition by intervals, we need no less than nine intervals, which are bounded by the discontinuity points of the interval, plus the discontinuity point of $h$ and its two preimages by $h$.

Remark 22. We denote the discontinuity point of $h$ by $\beta_{2}=\frac{\alpha+\alpha^{2}}{2}$, its preimage by $\beta_{1}=\frac{\alpha^{2}+\alpha^{4}}{2}$, and its second preimage by $\beta_{3}=\alpha+\frac{\alpha^{2}+\alpha^{3}}{2}$, or $\beta_{0}=-\frac{\alpha^{2}+\alpha^{3}}{2}=\beta_{3}$ ( $\left.\bmod 1\right)$. These last three points are the images by $T$ of the points $0, \alpha, \alpha^{2}$, and the opposite on the circle of the other discontinuity points; they are discontinuity point for the inverse map $T^{-1}$.

Let $\mathcal{A}=\{1, \ldots, 9\}$. We consider the partition

$$
\mathcal{I}=\left\{I_{i}, i \in \mathcal{A}\right\}
$$




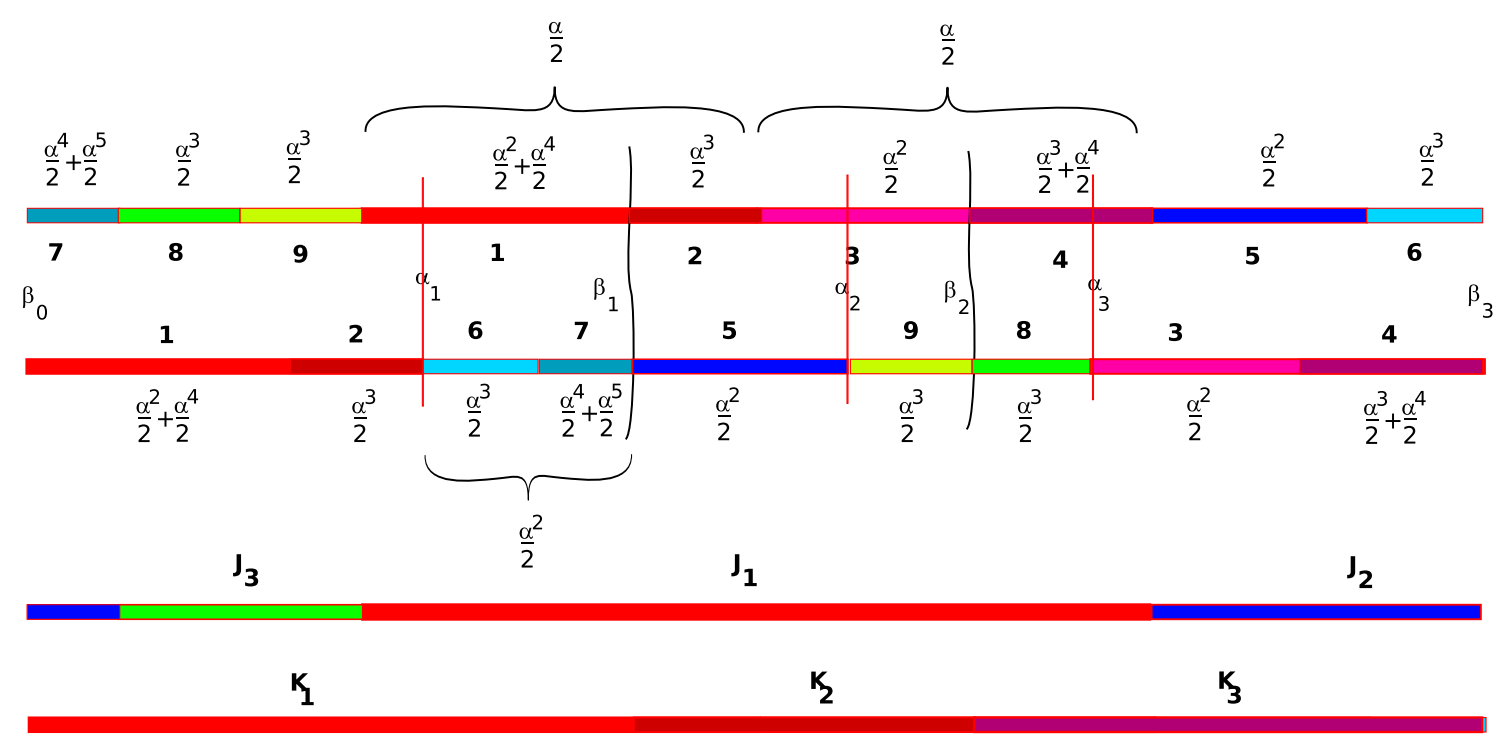

Figure 1. The partitions of the interval.

of the circle $\mathbb{S}^{1}$ into 9 successive intervals (left closed, right open), with $I_{1}=\left[0, \frac{\alpha^{2}+\alpha^{4}}{2}\right.$ ) and of respective lengths

$$
\frac{\alpha^{2}+\alpha^{4}}{2}, \frac{\alpha^{3}}{2}, \frac{\alpha^{2}}{2}, \frac{\alpha^{3}+\alpha^{4}}{2}, \frac{\alpha^{2}}{2}, \frac{\alpha^{3}}{2}, \frac{\alpha^{4}+\alpha^{5}}{2}, \frac{\alpha^{3}}{2}, \frac{\alpha^{3}}{2} .
$$

This partition is defined by the six discontinuity points $0, \alpha / 2, \alpha, \alpha+\alpha^{2} / 2, \alpha+\alpha^{2}, \alpha+$ $\alpha^{2}+\alpha^{3} / 2$ of $T$ (on the circle) and by the three points $\beta_{i}$ (defined in Remark 22). It is a refinement of the natural partition by the continuity intervals; hence $T$ can also be viewed as an interval exchange map on this partition.

In what follows, it will be more convenient for computations to consider $T$ as defined on an interval. It turns out to be inconvenient to split the circle at 0 , because this introduces a new discontinuity. But the points $\beta_{n}$ are images of discontinuity points for $T$; hence, if we split the circle at $\beta_{i}$, we get a better model. From now on, we will consider $T$ as defined on the interval $\left[\beta_{0}, \beta_{3}\right)$. This is the convention used in the figure 1 , this is why the intervals appear in the order $(7,8,9,1,2,3,4,5,6)$, and their images in the order $(1,2,6,7,5,9,8,3,4)$. The translation vector is given by:

$$
t=\frac{1}{2}\left(\alpha-1, \alpha-1,1-\alpha, 1-\alpha, \alpha^{2}-1,-1-\alpha^{2}, 1-\alpha^{2}, 2-\alpha-\alpha^{2}, \alpha+\alpha^{2}\right) .
$$

We will denote by $t_{i}$ the components of $t$.

The partition $\mathcal{I}$ is finer than the partition $\mathcal{J}$ defined above, since $J_{a}=I_{1} \cup I_{2} \cup I_{3} \cup I_{4}$, $J_{b}=I_{5} \cup I_{6} \cup I_{7}, J_{c}=I_{8} \cup I_{9}$. It is also finer than the partition by continuity intervals, since $I_{1} \cup I_{2}, I_{3} \cup I_{4}$ and $I_{6} \cup I_{7}$ are continuity intervals.

We define another partition $\mathcal{K}$ by

$$
\begin{gathered}
\mathcal{K}=\left\{K_{1}, K_{2}, K_{3}\right\}=\left\{I_{7} \cup I_{8} \cup I_{9} \cup I_{1}, I_{2} \cup I_{3}, I_{4} \cup I_{5} \cup I_{6}\right\} \\
=\left\{T\left(I_{1}\right) \cup T\left(I_{2}\right) \cup T\left(I_{6}\right) \cup T\left(I_{7}\right), T\left(I_{5}\right) \cup T\left(I_{9}\right), T\left(I_{8}\right) \cup T\left(I_{3}\right) \cup T\left(I_{4}\right)\right\},
\end{gathered}
$$




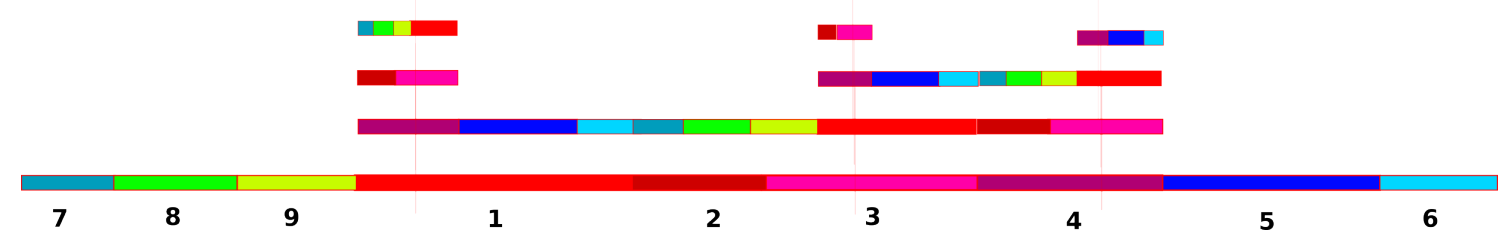

Figure 2. The induction

so that $K_{1}=\left[\beta_{0}, \beta_{1}\right), K_{2}=\left[\beta_{1}, \beta_{2}\right)$ and $K_{3}=\left[\beta_{2}, \beta_{3}\right)$. The length of these intervals are $\left|K_{1}\right|=\frac{\alpha+\alpha^{2}}{2}$ (contains the origin), $\left|K_{2}\right|=\frac{\alpha^{2}+\alpha^{3}}{2}$, and $\left|K_{3}\right|=\frac{\alpha+\alpha^{3}}{2}$. As we will see below, that partition plays an important role in the induction.

Definition 23. We denote by $k^{-}$and $k^{+}$the two maps from the alphabet $\mathcal{A}$ to $\{1,2,3\}$ defined by $I_{i} \subset K_{k^{-}(i)}$ and $T\left(I_{i}\right) \subset K_{k^{+}(i)}$.

Finally we consider the partition $\hat{\mathcal{J}}=\mathcal{J} \vee \mathcal{K}$ :

$$
\hat{\mathcal{J}}=\left\{\hat{J}_{1}, \hat{J}_{2}, \hat{J}_{4}, \hat{J}_{5}, \hat{J}_{7}, \hat{J}_{9}\right\}=\left\{I_{1}, I_{2} \cup I_{3}, I_{4}, I_{5} \cup I_{6}, I_{7}, I_{8} \cup I_{9}\right\}
$$

which is indexed by the alphabet $\hat{\mathcal{A}}=\{1,2,4,5,7,8\} \subset \mathcal{A}$. This partition will arise naturally in geometrical constructions of subsections 6.1 and 6.2 .

3.1.3. McMullen's curve. The map $T$ is algebraic, hence it allows the construction explained in Subsection 2.6.2. This construction will be detailled in Subsection 6.3. For now, observe that all lengths of the intervals of $T$ belong to $\frac{1}{2} \mathbb{Z}[\alpha]$. We can map bijectively $\mathbb{Z}^{3}$ onto $\frac{1}{2} \mathbb{Z}[\alpha]$ by the map :

$$
Q:(n, p, q) \mapsto \frac{n}{2}+\frac{p}{2} \alpha+\frac{q}{2} \alpha^{2} .
$$

We observe that $\mathcal{Z}=Q^{-1}\left(\frac{1}{2} \mathbb{Z}[\alpha] \cap\left[\beta_{0}, \beta_{3}\right)\right)$ is a "slice" in $\mathbb{Z}^{3}$ between two planes. Note that for all $(p, q) \in \mathbb{Z}^{2}$ there is a unique $n$ such that $Q(n, p, q) \in\left[\beta_{0}, \beta_{3}\right), Q(n+1, p, q) \in\left[\beta_{0}, \beta_{3}\right)$, i.e. there are exactly two points of type $(n, p, q)$ for a fixed pair $(p, q)$ in the slice (the original curve was defined using the interval $[0,1)$ instead of $\left[\beta_{0}, \beta_{3}\right)$, but this would be less convenient).

We will call McMullen's curve the curve underlying the stepped line (denoted by $\mathcal{L}$ ) between successive points $\left(Q^{-1}\left(T^{n}\left(\alpha_{1}\right)\right)\right)_{n \geq 0}$ of the orbit of the fixed point seen in $\mathbb{Z}^{3}$, see the figure in [14]. It is a stepped line since the differences $Q^{-1}\left(T^{n+1}\left(\alpha_{1}\right)\right)-Q^{-1}\left(T^{n}\left(\alpha_{1}\right)\right)$ are given by the $Q^{-1}\left(t_{i}\right)$, so they only take a finite number of values.

\subsection{The substitution.}


3.2.1. Definition. We introduce the substitution $\sigma$ on the alphabet $\mathcal{A}$

$$
\begin{aligned}
\sigma: & 1 \rightarrow 35 \\
2 & \rightarrow 45 \\
3 & \rightarrow 46 \\
4 & \rightarrow 17 \\
5 & \rightarrow 18 \\
6 & \rightarrow 19 \\
7 & \rightarrow 29 \\
8 & \rightarrow 2 \\
9 & \rightarrow 3 .
\end{aligned}
$$

The substitution $\sigma$ has a periodic orbit of period 3. Set $u=u^{(1)}=\lim _{n \rightarrow \infty} \sigma^{3 n}(1), u^{(2)}=$ $\lim _{n \rightarrow \infty} \sigma^{3 n}(3)$ and $u^{(3)}=\lim _{n \rightarrow \infty} \sigma^{3 n}(4)$; the periodic orbit is $u^{(1)} \stackrel{\sigma}{\rightarrow} u^{(2)} \stackrel{\sigma}{\rightarrow} u^{(3)} \stackrel{\sigma}{\rightarrow} u^{(1)}$. It has no other periodic orbit.

\subsubsection{Dynamics.}

Proposition 24. The dynamical system $\left(\mathbb{S}^{1}, T\right)$ has $\sigma$-structure with respect to the partition $\mathcal{I}$.

Proof. This expresses the induction scheme. We know that the map $T$ is conjugate by $h$ to its induced map on $J_{1}=I_{1} \cup I_{2} \cup I_{3} \cup I_{4}$. We have to check the $\sigma$-structure. But a simple computation shows that $h\left(I_{1}\right) \subset I_{3}, T\left(h\left(I_{1}\right)\right) \subset I_{5}$, and $T^{2}\left(h\left(I_{1}\right)\right) \subset J_{1}$, hence the itinerary of the interval $h\left(I_{1}\right)$ is 35 ; similar computations for the other intervals show that we have a $\sigma$-structure for the substitution $\sigma$ above.

Since the elements of the partition $\mathcal{I}$ are continuity intervals for the minimal map $T$, the partition is generating, that is, the map $\varphi_{\mathcal{I}}$ which sends each point to its itinerary with respect to the partition $\mathcal{I}$ is one-to-one. And since we have a $\sigma$-structure, the coding of the IET $T$ is well decribed by the substitutive dynamical system $(\Omega, S)$, where $S$ denotes the usual shift, and the following diagram commutes:

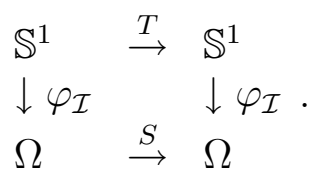

Similarly, the following diagram, which expresses the induction, commutes :

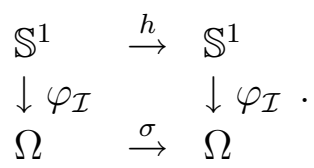

Remark 25. It is interesting, to better see the induction, to define the interval exchange on the disjoint union of the three intervals $K_{1}, K_{2}$ and $K_{3}$, as suggested by the figure 2 .

Indeed, we can check that the conjugacy map $h$ defined above satisfies $h\left(K_{1}\right)=K_{2}$, $h\left(K_{2}\right)=I_{4} \subset K_{3}$ and $h\left(K_{3}\right)=I_{1} \subset K_{1}$.

We can take local coordinates on each $K_{i}$ by taking the distance to the periodic point $\alpha_{i}$; more precisely, we define a map $\left[\beta_{0}, \beta_{3}\right) \rightarrow \mathbb{R} \times \mathbb{Z} / 3 \mathbb{Z}, t \mapsto\left(t-\alpha_{i}, i\right)$ if $x \in K_{i}$. A direct computation shows that, in these coordinates, $h$ can be written $h(x, i)=(\alpha x, i+1)$; 
the cube $h^{3}$ of $h$ is an homothety on each interval $K_{i}$, centered in $\alpha_{i}$, as can be seen in the figure 2.

Observe that the periodic orbit of h corresponds to the periodic orbit of $\sigma$ in the following way:

$$
\varphi_{\mathcal{I}}\left(\alpha_{1}\right)=u, \varphi_{\mathcal{I}}\left(\alpha_{2}\right)=u^{(2)}, \text { and } \varphi_{\mathcal{I}}\left(\alpha_{3}\right)=u^{(3)} .
$$

We will see that this periodic structure of order 3 of the induction appears in the spectral theory for the substitution $\sigma$.

3.2.3. Factors. There are two natural factors of this symbolic dynamical system.

The first one follows from the identification $\rho: \mathcal{A} \rightarrow \breve{\mathcal{A}}=\{a, b, c\}$ defined by $I_{i} \subset J_{\rho(i)}$, i.e. $1,2,3,4 \mapsto a ; 5,6,7 \mapsto b ; 8,9 \mapsto c$. The map $\rho$ defines an equivalence relation on the alphabet, which extends to the free monoid $\mathcal{A}^{*}$ : two words are equivalent if they have the same image by $\rho$. One checks immediately that this equivalence relation is compatible with the substitution $\sigma$, and the factor substitution is the Tribonacci substitution defined above in Example 4:

$$
\begin{aligned}
\breve{\sigma}: a & \rightarrow a b \\
b & \rightarrow a c \\
c & \rightarrow a .
\end{aligned}
$$

The other one follows from the identification $2 \cong 3,5 \cong 6$ and $8 \cong 9$ and corresponds to the coding by the partition $\hat{\mathcal{J}}$. This equivalence relation is also compatible with the substitution, and the factor substitution, on the alphabet $\hat{\mathcal{A}}=\{1,2,4,5,7,8\}$ is

$$
\begin{aligned}
\hat{\sigma}: & 1 \rightarrow 25 \\
2 & \rightarrow 45 \\
4 & \rightarrow 17 \\
5 & \rightarrow 18 \\
7 & \rightarrow 28 \\
8 & \rightarrow 2 .
\end{aligned}
$$

Observe that the equivalence relation corresponding to coding by partition $\mathcal{K}$ is not compatible with $\sigma$ : letters 1 and 9 are equivalent for this partition, and their image do not even have the same length, hence there is no factor substitution for this partition.

3.2.4. Linear algebra. The matrix $M_{\sigma}$ associated with $\sigma$ is a $9 \times 9$ matrix, whose characteristic polynomial is $P(X)=X^{9}-X^{7}-5 X^{6}-X^{5}+X^{4}+5 X^{3}+X^{2}-1$, which factorizes into

$$
P(X)=(X-1)\left(X^{2}+X+1\right)\left(X^{3}+X^{2}+X-1\right)\left(X^{3}-X^{2}-X-1\right) .
$$

The Perron-Frobenius eigenvalue is $\lambda=1.8393 \cdots$, the real root of $X^{3}-X^{2}-X-1$. We decompose the space $\mathbb{R}^{\mathcal{A}}$ into three invariant subspaces of dimension three with rational equation corresponding to this factorization, $\mathbb{R}^{\mathcal{A}}=E_{n} \oplus E_{T} \oplus E_{\bar{T}}$, where

- The invariant subspace $E_{n}$ is associated with the eigenvalues $1, j, j^{2}$; it splits into $E_{n}=E_{I} \oplus E_{j}$ where $E_{I}$ is the eigenspace associated with eigenvalue 1 , and $E_{j}$ is the invariant plane associated with the pair of eigenvalues $j, j^{2}$.

- The invariant subspace $E_{\bar{T}}=\operatorname{ker}\left(M^{3}+M^{2}+M-I d\right)$, corresponds to eigenvalues $\alpha<1, \beta$ and $\bar{\beta}$, roots of $X^{3}+X^{2}+X-1$ and splits into $E_{\bar{T}}=E_{\bar{T}, s} \oplus E_{\bar{T}, u}$ a contracting direction and an expanding plane. 
- The invariant subspace $E_{T}=\operatorname{ker}\left(M^{3}-M^{2}-M-I d\right)$, corresponds to eigenvalues $\lambda>1, \mu$ and $\bar{\mu}$ also splits into $E_{T}=E_{T, u} \oplus E_{T, s}$, the Perron-Frobenius direction and the contracting plane.

The space $E_{\bar{T}, u} \oplus E_{T, u}$ is the invariant unstable space $E_{u}$, and the space $E_{\bar{T}, s} \oplus E_{T, s}$ is the invariant stable space $E_{s}$. We summarize

$$
\begin{aligned}
\mathbb{R}^{\mathcal{A}} & =E_{n} \oplus E_{\bar{T}} \oplus E_{T}=E_{n} \oplus E_{s} \oplus E_{u} \\
& =E_{I} \oplus E_{j} \oplus E_{\bar{T}, s} \oplus E_{\bar{T}, u} \oplus E_{T, u} \oplus E_{T, s}
\end{aligned}
$$

If $A=\left(\begin{array}{ccc}1 & 1 & 1 \\ 1 & 0 & 0 \\ 0 & 1 & 0\end{array}\right)$ denotes the Tribonacci matrix, and $J=\left(\begin{array}{ccc}0 & 0 & 1 \\ 1 & 0 & 0 \\ 0 & 1 & 0\end{array}\right)$, the permutation matrix then the factorization of the characteristic polynomial implies that we can find a rational matrix $P$ such that the conjugate matrix $\widetilde{M}$ is given by

$$
\widetilde{M}=P^{-1} M P=\left(\begin{array}{rrr}
J & 0 & 0 \\
0 & { }^{t} A^{-1} & 0 \\
0 & 0 & A
\end{array}\right)
$$

We denote by $\Pi_{*}$ the projection onto $E_{*}$ parallel to the supplementary space according to decomposition (3), where $E_{*}$ is any invariant subspace. This defines a family of projection $\Pi_{n}, \Pi_{T}, \Pi_{T, s} \ldots$, and we will mostly consider these projections (except in some particular cases, where we will make explicit our choice of projection) and use them to define stepped lines.

\section{StABLE}

In this part, we concentrate on what happens in the stable subspace $E_{s}$. In stable directions, the Rauzy fractal construction procedure is natural. We will see that it works well if we restrict to the two-dimensional space $E_{T, s}$ but that the situation is more intricated in the direction $E_{\bar{T}, s}$. To obtain a nicer picture, we will involve the neutral direction, and recover in a systematic way the interval exchange from the substitution, and a Peano curve.

4.1. Projection to the Tribonacci stable plane. The equivalence relation defined on $\mathcal{A}$ by the partition $\mathcal{J}$ defines a subspace of dimension 6. A small computation shows that this space is $E_{n} \oplus E_{\bar{T}}$; the projection $\Pi_{T}$ on the space $E_{T}$ satisfies by construction $\Pi_{T}\left(e_{1}\right)=\Pi_{T}\left(e_{2}\right)=\Pi_{T}\left(e_{3}\right)=\Pi_{T}\left(e_{4}\right), \Pi_{T}\left(e_{5}\right)=\Pi_{T}\left(e_{6}\right)=\Pi_{T}\left(e_{7}\right), \Pi_{T}\left(e_{8}\right)=\Pi_{T}\left(e_{9}\right)$. If we define $e_{a}=\Pi_{T}\left(e_{1}\right), e_{b}=\Pi_{T}\left(e_{5}\right), e_{c}=\Pi_{T}\left(e_{8}\right)$, we obtain a basis of the 3 -dimensional space $E_{T}$, which can be seen as $\mathbb{R}^{\breve{A}}$, on which the restriction of $M$ acts as $A$.

The canonical stepped line $\mathbf{L}^{\breve{u}}$ associated with the Tribonacci substitution can be embedded in this way in $E_{T}$, and we have the following result, which is not surprising in view of [1]:

\section{Theorem 26.}

$$
\Pi_{T}\left(\boldsymbol{L}^{u^{(i)}}\right)=\boldsymbol{L}^{\breve{u}} \text { for } i=1,2,3 .
$$



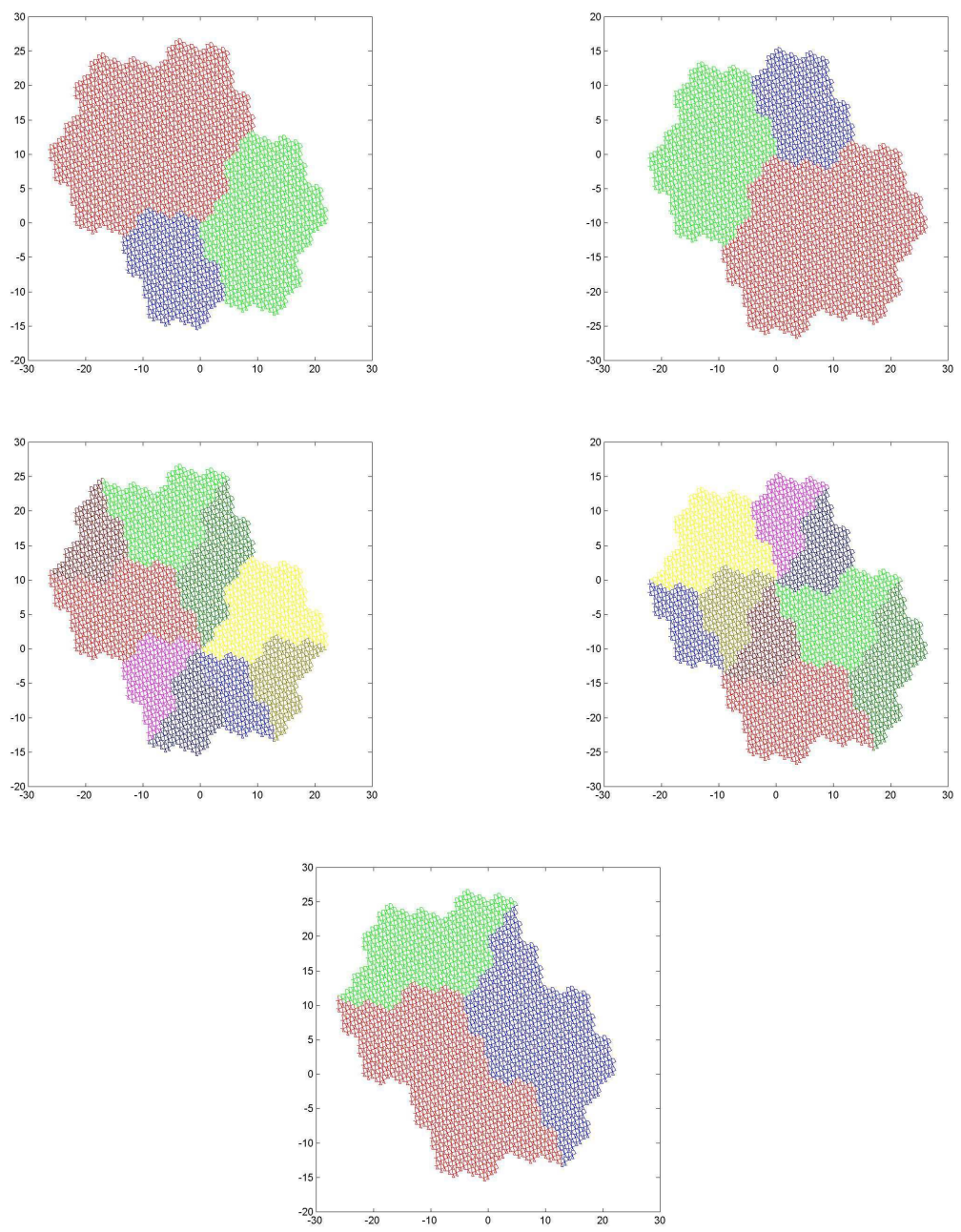

Figure 3. The Tribonacci fractal. Partition $\mathcal{J}$ (standard Tribonacci) before and after exchange, partition $\mathcal{I}$, before and after exchange and partition $\mathcal{K}$.

Proof. This result is a straightforward consequence of the analysis done in the previous section. Indeed, the projection $\rho$ from $\mathcal{A}$ to $\mathcal{A}$ sends the three periodic points $u^{(i)}$ of $\sigma$ to the fixed point $\breve{u}$ of $\breve{\sigma}$, so the corresponding projection $\Pi_{T}$ sends the three corresponding canonical stepped lines to the canonical stepped line $\mathbf{L}^{\breve{u}}$ in $E_{T}=\mathbb{R}^{\breve{A}}$.

As an immediate consequence, we see that

\section{Corollary 27.}

$$
\overline{\Pi_{T, s}\left(\mathcal{V}^{u}\right)}=\breve{\mathcal{R}} .
$$

The vertices of the stepped line project on the Tribonacci fractal. The partition $\left\{\Pi_{T, s}\left(\mathcal{V}^{u, a}\right), a \in \mathcal{A}\right\}$ (up to zero measure sets) is shown on Figure 3 before and after the exchange of pieces. The standard partition of the Tribonacci fractal is obtained by taking the unions of tiles with same $\rho$. 


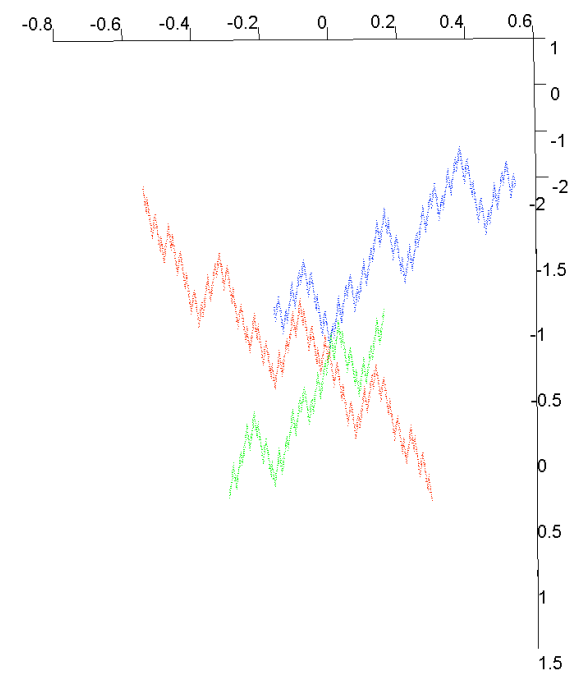

Figure 4. A view of $\Pi_{s}\left(\mathcal{V}^{u}\right)$. The colors correspond to partition $\mathcal{K}$. Note that this is a two dimensional view of a 3-dimensional curve with high Hausdorff dimension; this picture is to be related with Figures 5 and 6.

Remark 28. Observe that the same result holds for the stepped lines associated with the other fixed points since these fixed points also project onto $\breve{u}$. This may seem paradoxical at first glance since, naturally, the three fixed points are different though they yield the same partition. An explanation could be that the partition is up to a set of zero measure: indeed, the sets $\Pi_{T, s}\left(\mathcal{V}^{u^{(1)}, a}\right)$ and $\Pi_{T, s}\left(\mathcal{V}^{u^{(2)}, a}\right)$ differ on their boundaries, while the set of indices on which the corresponding fixed points are distinct can be of zero density.

4.2. Projection to the stable direction in AntiTribonacci. A straightforward application of the results of subsection 2.4 shows that the sets $\Pi_{\bar{T}}\left(\mathcal{V}^{u, a}\right)$ are compact sets in $E_{\bar{T}, s}$. They satisfy a GIFS, which can be written explicitly, using the fact that, with notation introduced in Section 3.1.2, $\Pi_{\bar{T}, s}\left(e_{i}\right)=t_{i}-\left(\alpha_{k^{+}(i)}-\alpha_{k^{-}(i)}\right)$.

This GIFS can be solved explicitly, and the result is slightly messy: the adherences of the $\Pi_{\bar{T}}\left(\mathcal{V}^{u, a}\right)$ are overlapping intervals. More precisely, we get copies of the intervals $K_{1}, K_{2}, K_{3}$, each centered at $\alpha_{i}$, since these points correspond to the three periodic points and project to 0 ; hence they overlap.

We will not go into further details, since a clearer picture will emerge in the following sections.

4.3. Projection on the complete stable space. We can gather the results of the two previous subsections by considering the projection $\Pi_{s}$ on the space $E_{s}$ parallel to $E_{u} \oplus E_{n}$. We are interested in $\Pi_{s}\left(\mathbf{L}^{u}\right)$. For the same reasons as in Section 4.2, the picture is still a little bit messy. But here, there is no overlap because the component $E_{T, s}$ separates points that would have the same projection on $E_{\bar{T}, s}$. Indeed, the projection is the union of three continuous (fractal) curves, as shown on Figure 4. Proof of such statement would follow from Theorem 30 which makes explicit the complete picture. 
4.4. Recovering the IET: a first attempt using another projection to $E_{\bar{T}, s}$. We use the results of subsection 2.6, and consider the projection $\Pi_{/}$onto $E_{\bar{T}, s}$ parallel to the hyperplane $H$ of equation $\sum_{i=1}^{d} t_{i} x_{i}=0$.

We observe that the subspace $H$ is related to the eigenspaces as follows: $E_{T} \subset H$, $E_{\bar{T}, u} \subset H, E_{I} \subset H$ and $H \oplus E_{\bar{T}, s}=\mathbb{R}^{\mathcal{A}}$; the relation between $H$ and $E_{j}$ will appear later.

We are interested in the projection of $\mathcal{V}^{u}$. The following theorem is an immediate consequence of the result of Subsection 2.6, since the projections of the basis vectors are the translation lengths of the interval exchange map $T$.

Theorem 29. $\overline{\Pi /\left(\mathcal{V}^{u}\right)}$ is an interval. The subsets $\overline{\Pi /\left(\mathcal{V}^{u, a}\right)}$ are intervals with disjoint interiors and the exchange of pieces is homothetic to the original map $T$.

As we will see below, what we did by changing the projection was just to make a constant translation on each copy of the $K_{i}$ to remove the overlap.

4.5. The Tribonacci filling curve. We want to extend the projection $\Pi_{/}$to all of $E_{s}$. Recalling $E_{T, s} \subset H$, we define the projection $\Pi_{\widetilde{s}}$ on the space $E_{s}$ parallel to $\tilde{H}=$ $H \cap\left(E_{u} \oplus E_{n} \oplus E_{\bar{T}, s}\right)$. We can write $\Pi_{\widetilde{s}}=\Pi_{T, s} \oplus \Pi_{/}$. We are interested in $\Pi_{\widetilde{s}}\left(\mathcal{V}^{u}\right)$. This object lies in $E_{s}=E_{T, s} \oplus E_{\bar{T}, s}$ which we can see as $\mathbb{R}^{3}$.

Theorem 30. $\overline{\Pi_{\widetilde{s}}\left(\mathcal{V}^{u}\right)}$ is a continuous curve in $\mathbb{R}^{3}$. Its projection on $E_{T, s}$ parallel to $E_{\bar{T}, s}$ is a Peano curve filling the Tribonacci fractal $\breve{\mathcal{R}}$. Its projection on $E_{\bar{T}, s}$ parallel to $E_{T, s}$ is one-to-one, and its image is an interval.

In other words, $\overline{\Pi_{\widetilde{s}}\left(\mathcal{V}^{u}\right)}$ is the graph of a Peano curve filling the Tribonacci fractal $\breve{\mathcal{R}}$ parameterized by the coordinate along $E_{\bar{T}, s}$.

Proof. Basically this is a consequence of Theorem 26 and Theorem 29. Continuity follows from the fact that if two points are close in direction $E_{\bar{T}, s}$, they are (except for a finite number of orbits) close symbolically and hence close in $E_{T}$. More specifically, if $\Pi /\left(x_{n}^{u}\right)$ is a point which does not belong to the orbits of boundary points of the intervals $I_{i}$, for any $N>0$ there is a neigborhood $V$ of $\Pi /\left(x_{n}^{u}\right)$ such that all points in $V$ have the same dynamics for $N$ steps, so that any other $x_{m}^{u}$ with $\Pi_{/}\left(x_{m}^{u}\right) \in V$ will satisfy $\Pi_{T, s}\left(x_{m}^{u}\right)$ close to $\Pi_{T, s}\left(x_{n}^{u}\right)$. So that all we have to check is continuity at the boundaries of the atoms of the partition $\mathcal{I}$. Since it projects on $E_{T}$, the only delicate points are the boundaries of $J_{a}, J_{b}$ and $J_{c}$. But they all project on the same point.

Observe that the points corresponding to the prefixes of the fixed point appear on the curve in an order prescribed by the dynamics, which is in a sense dual to the order in which they are disposed on the $E_{\bar{T}, s}$ axis. It is a straightforward way to see the Peano curve in the Tribonacci fractal constructed by Arnoux in [1] (the proof in this paper is essentially the one we just gave; the essential step is to prove that discontinuity points have the same image). Indeed, it defines a map from the interval (or the circle $\mathbb{S}^{1}$ ) to the Tribonacci fractal. Observe that the sets $\overline{\Pi_{\widetilde{s}}\left(\mathcal{V}^{u, a}\right)}$ project onto compact disjoint (up to boundary points) pieces of the Tribonacci fractal.

The fact that the projection of the stepped line is a curve is far from obvious from the construction, since we just project a discrete set in $\mathbb{R}^{\mathcal{A}}$. We will see later another way to construct this Peano curve as a limit of a sequence of renormalized stepped lines, associated with the so called dual substitution, which gives a curve by construction. 

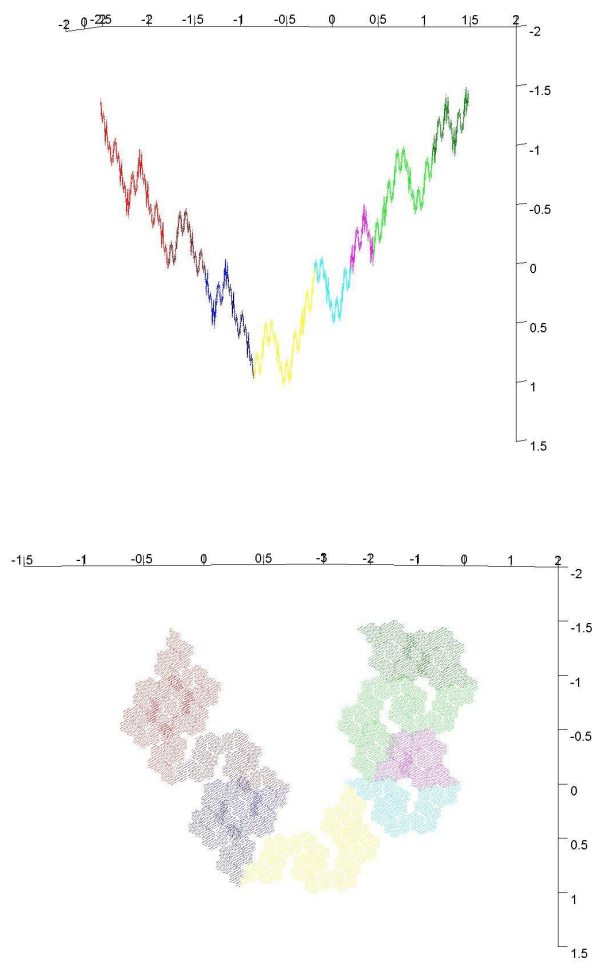
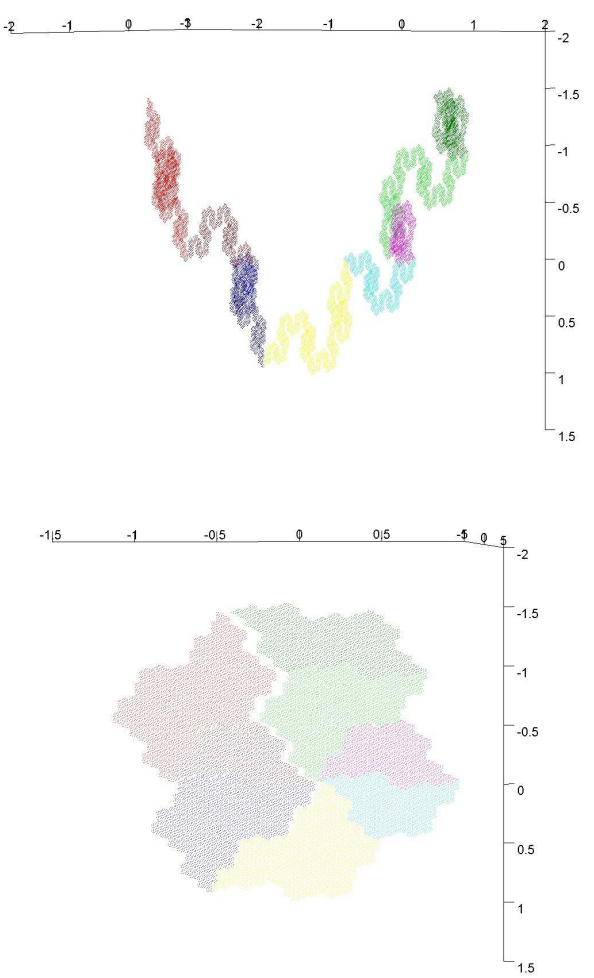

Figure 5. Different views of the Tribonacci filling curve $\Pi_{\widetilde{s}}\left(L^{u}\right)$. The colours correspond to the partition $\left\{\overline{\Pi_{\widetilde{s}}\left(\mathcal{V}^{u, a}\right)}, a \in A\right\}$.

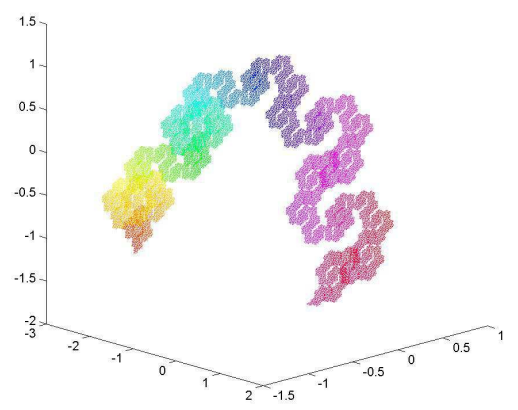

Figure 6. A view of the Tribonacci filling curve $\Pi_{\widetilde{s}}\left(L^{u}\right)$. Here colours continuously change along coordinate $E_{\bar{T}, s}$.

\section{NEUTRAL}

We now study in a more detailled way the dynamical behaviour in the neutral subspace $E_{n}$. In general, the projections on the neutral space could be quite complicated, since we cannot expect convergent series and there can be a unbounded drift. However, in our case, some remarkable coincidences occur, and things are quite simple and almost completely understood. Indeed, the space $E_{n}$ splits into $E_{j} \oplus E_{I}$. In $E_{j}$, the Rauzy fractal procedure 


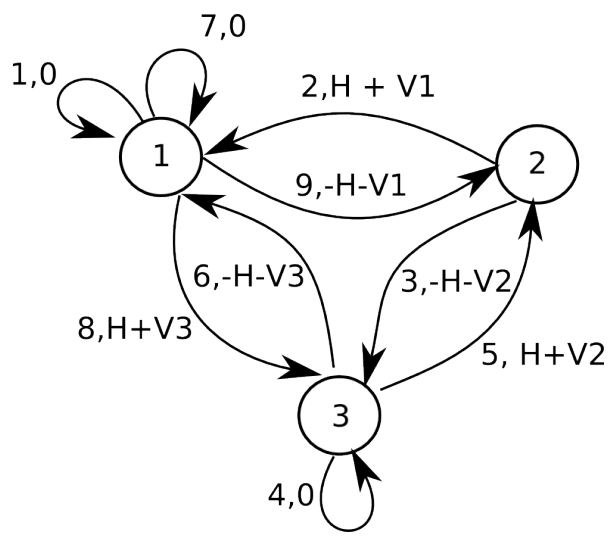

FiguRE 7. Graph of the approximating subshift : vertices are 1,2 and 3 and edges are labelled by $\mathcal{A}$ and $\left\{\Pi_{j}\left(e_{a}\right), a \in \mathcal{A}\right\}$, with $H=\Delta / 3$.

yields a finite set. Along $E_{I}$, the stepped line projects onto a discrete but unbounded set. We use this result to give another formulation of Theorem 29 making more clear the role of the partition $\mathcal{K}$.

5.1. Projection to the neutral subspace. We first analyze the projection of $\mathbf{L}^{u}$ onto the space $E_{j}$. Let $M_{1}=0, M_{2}=-\Pi_{j}\left(e_{2}\right), M_{3}=\Pi_{j}\left(e_{8}\right)$ and set $\mathcal{M}=\left\{M_{1}, M_{2}, M_{3}\right\}$.

Theorem 31. The projection of $\mathcal{V}^{u}$ onto $E_{j}$ is reduced to $\mathcal{M}$.

Proof. Computation (see Section 9) shows that $\Pi_{j}\left(e_{1}\right)=\Pi_{j}\left(e_{4}\right)=\Pi_{j}\left(e_{7}\right)=0$, that $\Pi_{j}\left(e_{2}\right)=-\Pi_{j}\left(e_{9}\right), \Pi_{j}\left(e_{3}\right)=-\Pi_{j}\left(e_{5}\right), \Pi_{j}\left(e_{6}\right)=-\Pi_{j}\left(e_{8}\right)$, and that $\Pi_{j}\left(e_{2}+e_{8}+e_{5}\right)=0$.

Consider the graph $G$ (see Figure 7 ) with vertices $\{1,2,3\}$ and for which there is an edge from $r$ to $s$ labelled $\left(a, \Pi_{j}\left(e_{a}\right)\right)$ if and only if $k^{-}(a)=r$ and $k^{+}(a)=s$ (where $k^{-}$and $k^{+}$have been defined in Definition 23). Remark that, for any $a \in \mathcal{A}$, there is exactly one edge labeled by $\left(a, \Pi_{j}\left(e_{a}\right)\right)$. Any itinerary $\varphi_{\mathcal{I}}(x)=\left(v_{n}\right)_{n \in \mathbb{N}}$ is the label of a path in this graph, since, by definition, we must have $k^{-}\left(v_{n+1}\right)=k^{+}\left(v_{n}\right)$; the corresponding sequence of vertices is just the itinerary $\varphi_{\mathcal{K}}(x)$ of $x$ with respect to the partition $\mathcal{K}$.

This is true in particular for the fixed point $u$; moreover, since $u_{0}=1$, we have $I_{u_{0}} \subset K_{1}$ and the corresponding path starts from vertex 1 .

One checks immediately that, for all $a \in \mathcal{A}, \Pi_{j}\left(e_{a}\right)=M_{k^{+}(a)}-M_{k^{-}(a)}$; hence, the cocycle is a coboundary, and we have, for any itinerary $v$,

$$
\sum_{j=0}^{n} \Pi_{j}\left(e_{v_{j}}\right)=M_{k^{+}\left(v_{n}\right)}-M_{k^{-}\left(v_{0}\right)},
$$

in particular, for the fixed point, $M_{k^{-}\left(v_{0}\right)}=0$, so the projection of the corresponding canonical stepped line $\Pi_{j}\left(x_{n}^{u}\right)$ is in $\mathcal{M}$ for all $n \geq 1$, which proves the theorem.

The projection to the whole space $E_{n}$ is slightly more complicated. For convenience, we denote by $V_{1}$ (resp. $\left.V_{2}, V_{3}\right)$ the vector $\Pi_{j}\left(e_{2}\right)$ (resp. $\Pi_{j}\left(e_{5}, \Pi_{j}\left(e_{8}\right)\right)$, and by $\Delta=$ $-e_{1}+e_{3}-e_{5}+e_{6}-e_{8}+e_{9}$ the invariant vector (eigenvector for the eigenvalue 1); we have: 
Proposition 32. The projection of $\mathcal{V}^{u}$ onto $E_{n}$ is a discrete but unbounded subset of $\mathcal{M}+\mathbb{Z} \Delta / 3$, namely,

$$
\Pi_{n}\left(\mathcal{V}^{u}\right)=\left(M_{1}+\mathbb{Z} \Delta\right) \cup\left(M_{2}+\Delta / 3+\mathbb{Z} \Delta\right) \cup\left(M_{2}+2 \Delta / 3+\mathbb{Z} \Delta\right) .
$$

Remark 33. It is possible to prove that the drift in the $\Delta$-direction is logarithmic. We have no geometric interpretation for what happens in this direction.

Proof. Computation shows that $\Pi_{n}\left(e_{2}+e_{8}+e_{5}\right)=\Delta$ and $\Pi_{n}\left(e_{3}+e_{6}+e_{9}\right)=-\Delta$. Using the notations $V_{1}, V_{2}, V_{3}$ above, we compute the projections of the basis vectors on $E_{n}$ :

$$
\begin{aligned}
& \Pi_{n}\left(e_{1}\right)=\Pi_{n}\left(e_{4}\right)=\Pi_{n}\left(e_{7}\right)=0 \\
& \Pi_{n}\left(e_{2}\right)=V_{1}+\frac{\Delta}{3}=-\Pi_{n}\left(e_{9}\right) \\
& \Pi_{n}\left(e_{5}\right)=V_{2}+\frac{\Delta}{3}=-\Pi_{n}\left(e_{3}\right) \\
& \Pi_{n}\left(e_{8}\right)=V_{3}+\frac{\Delta}{3}=-\Pi_{n}\left(e_{6}\right) .
\end{aligned}
$$

Taking into account the $\Delta$-component, we immediately see that $\Pi_{n}\left(L_{n}^{u}\right) \subset \mathcal{M}+\mathbb{Z} \Delta / 3$. Let us first prove that the stepped line is unbounded in this direction. To do so we exhibit two sequences $W_{n}^{ \pm}$of prefixes of the fixed point $u$ for which $\Pi_{n}\left(e_{W_{n}^{ \pm}}\right)= \pm n \Delta / 2$. Indeed, consider $W_{1}^{-}=\sigma^{5}(3) \sigma^{3}(3) \sigma(3)$ and set for all $n \geq 1, W_{n+1}^{-}=\sigma^{6}\left(W_{n}^{-}\right) W_{1}^{-}$. It follows from a straightforward analysis of the prefix automaton that $W_{n}^{-}$are prefixes of the fixed point $u$. Since, $\Pi_{n}\left(M^{3} e_{i}\right)=\Pi_{n}\left(e_{i}\right)$,

$$
\Pi_{n}\left(e_{W_{n}^{-}}\right)=\Pi_{n}\left(\sum_{i=1}^{n} M^{6 n} e_{W_{1}^{-}}\right)=\sum_{i=1}^{n} \Pi_{n}\left(M^{6 n} e_{W_{1}^{-}}\right)=n \Pi_{n}\left(e_{W_{1}^{-}}\right) .
$$

Recalling that $W_{1}^{-}=\sigma^{5}(3) \sigma^{3}(3) \sigma(3)$, we conclude that

$$
\Pi_{n}\left(e_{W_{n}^{-}}\right)=n \Pi_{n}\left(M^{5} e_{3}+M^{3} e_{3}+M e_{3}\right)=n \Pi_{n}\left(e_{9}+e_{3}+e_{6}\right)=-n \Delta .
$$

In the same spirit, we consider $W_{0}^{+}=\sigma^{3}(1) \sigma^{2}(2), W_{1}^{+}=\sigma^{8}\left(W_{0}^{+}\right) \sigma^{4}\left(W_{0}^{+}\right) W_{0}^{+}$and set $W_{n+1}^{+}=\sigma^{12 n+6}\left(W_{0}^{+}\right) \sigma^{12 n}\left(W_{1}^{+}\right) \sigma^{12(n-1)}\left(W_{1}^{+}\right) \cdots \sigma^{12}\left(W_{1}^{+}\right) W_{1}^{+}$. Again, $W_{n}^{+}$is a prefix of $u$ and here,

$$
\Pi_{n}\left(e_{W_{n}^{+}}\right)=n \Delta .
$$

To conclude we observe that in view of the graph $G$, only points of the form $M_{i}+i \Delta / 3+n \Delta$ can be attained since moves in direction $\Delta$ correspond to period 3 cycles in the graph. The same analysis shows that all such point are attained since $n \Delta$ are attained for all $n \in \mathbb{Z}$.

5.2. Recovering the IET on three intervals: a more conceptual version. Here we project the stepped line $L^{u}$ onto $E_{j} \oplus E_{T, s}$. The Rauzy fractal we obtain is the union of three intervals that can be seen as the three intervals $K_{1}, K_{2}$ and $K_{3}$ of the partition $\mathcal{K}$. We recover Lemma 29 by projecting in a direction such that the three interval glue on a single segment. Denote $\Pi_{/ / /}$the natural projection onto $E_{j} \oplus E_{\bar{T}, s}$. Consider the set made of the union of three intervals, copies of $K_{1}, K_{2}, K_{3}$ parallel to $E_{\bar{T}, s}$ defined by $\widehat{K}=\left(M_{1}+\left(K_{1}-\alpha_{1}\right) e^{\bar{T}, s}\right) \cup\left(M_{2}+\left(K_{2}-\alpha_{2}\right) e^{\bar{T}, s}\right) \cup\left(M_{3}+\left(K_{3}-\alpha_{3}\right) e^{\bar{T}, s}\right)$. 

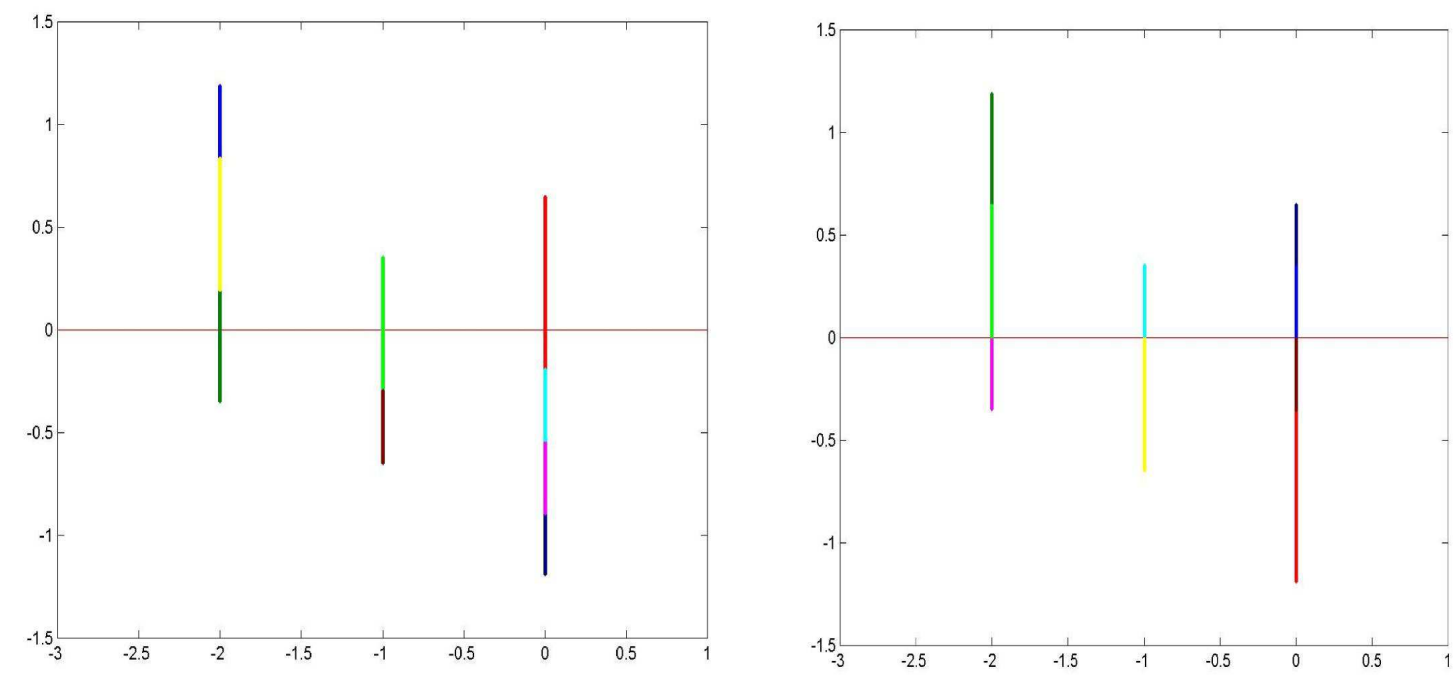

Figure 8. Projection on $E_{j} \oplus E_{T, s}$. Here $E_{j}$ is represented horizontally by only one dimension since the projection is reduced to 3 points.

Theorem 34. The adherence of the projection $\Pi_{/ / /}\left(\mathcal{V}^{u}\right)$ is equal to $\widehat{K}$. The subsets $\overline{\Pi_{/ / /}\left(\mathcal{V}^{u, a}\right)}$ are disjoint intervals (up to measure zero).

Proof. For $i \in \mathcal{A}$, let $k^{-}(i)$ be the index such that $I_{i} \subset K_{k^{-}(i)}$ and $k^{+}(i)$ such that $T\left(I_{i}\right) \subset K_{k^{+}(i)}$. It follows from analysis done in Section 5 that $\Pi_{j}\left(e_{i}\right)=M_{k^{+}(i)}-M_{k^{-}(i)}$, while, according to Lemma 57, $\Pi_{\bar{T}, s}\left(e_{i}\right)$ is proportionnal to $t_{i}-\alpha_{k^{+}(i)}+\alpha_{k^{-}(i)}$. Hence, the same argument as in the proof of Lemma 29 but considering the $K_{i}$ 's as disjoint intervals shows the result.

The interpretation is that the projection to the subspace $E_{j}$ corresponds to the segment $K_{i}$ containing the point, while the projection on $E_{\bar{T}, s}$ is proportionnal to distance of the point to the periodic point $\alpha_{i}$ (contained in $K_{i}$ ). This result stresses the importance of the partition we introduced, showing that the induction is better seen if we consider our map as defined on the union of the three intervals $K_{1}, K_{2}$ and $K_{3}$ instead of the circle. On the Rauzy fractal obtained here, the Abelianization matrix acts as a contraction/permutation, the three intervals being shrinked around $M_{i}$ and permuted.

\section{UNSTABLE}

As we saw in Subsection 2.5, in the unstable subspace $E_{u}$ there is no hope for constructing a Rauzy fractal since the projection of the stepped line is not bounded, but we can describe the behavior of the projection of the stepped line using a renormalisation procedure. The "renormalized" stepped line tends to a well identified Peano curve filling a planar domain (with geometric properties close to that of the Tribonacci fractal). Using properties of the neutral direction, we also give an interpretation of the McMullen curve as another projection of our stepped line, having the same asymptotic properties. 
6.1. Projection to the antiTribonacci unstable plane: the discrete stepped line. We are interested in the unbounded stepped lines $\Pi_{\bar{T}}\left(\mathbf{L}^{u}\right)$ and $\Pi_{\bar{T}, u}\left(\mathbf{L}^{u}\right)$. We are going first to describe some properties of these discrete stepped lines.

Theorem 35. The stepped line $\Pi_{\bar{T}}\left(\boldsymbol{L}^{u}\right)$ is unbounded but remains within bounded distance of the plane $E_{\bar{T}, u}$. It has multiple points of order not larger than 3 .

Proof. To see it is not bounded, it is enough to exhibit an unbounded sequence of points, for instance the $\Pi_{\bar{T}}\left(x_{\left|\sigma^{n}(1)\right|}^{u}\right)$. Since direction $E_{\bar{T}, s}$ is contracting, boundedness in the direction transversal to $E_{\bar{T}, u}$ is a consequence of Proposition 13. We observe that all points have integral coordinates (in a suitable basis). Hence they project to distinct points on the $E_{\bar{T}, s}$ (irrational) axis (parallel to $E_{\bar{T}, u}$ ). But no more than three points can have the same projection on $E_{\bar{T}, s}$ in view of Theorem 31 (one coming from each $K_{i}$ ); there are indeed multiple points of order up to 3.

Remark 36. We mention without proof that the projection on $E_{\bar{T}, u}$ of this stepped line has no crossings. It is quite easy to see that no crossing can occur at the multiple points (it suffices to analyse words of length two giving rise to multiple points). It is more difficult, but true, that two edges (between successive points) of the stepped line can not cross; this is done in the paper by Lowenstein et al., see [13], and we could not do better than duplicate their analysis.

The space $E_{n}$ admits as basis $\left\{e_{2}-e_{3}, e_{5}-e_{6}, e_{8}-e_{9}\right\}$; hence the projection on $E_{T} \oplus E_{\bar{T}}$ gives the same image to $e_{2}$ and $e_{3}, e_{5}$ and $e_{6}, e_{8}$ and $e_{9}$; the projection on the stepped line on this space is in fact the canonical stepped line associated with the substitution $\hat{\sigma}$. The projection on $E_{\bar{T}}$ of the canonical stepped line associated with $\sigma$ is also the projection of the stepped line associated with $\hat{\sigma}$.

When we draw the picture of the projection on $E_{\bar{T}, u}$ of the canonical stepped line associated with $u$, the picture, for a large number of points, seems to fill an approximate angular sector of the plane. The exact behavior can be understood completely; we give the results and omit the proof which is straightforward, but tedious.

We need first to consider the three infinite periodic points $u, u^{(2)}, u^{(3)}$; the corresponding stepped lines, which project almost to the same points in $E_{T}$, project to disjoint domains in $E_{\bar{T}}$, but this only fills half of the plane, in three disjoint angular sectors.

We also need to consider periodic left-infinite fixed points, indexed by negative integers. A small computation shows that there are 2 orbits of order 3 : one consists in left-infinite periodic points ending in 2,5,8, and the other in points ending in 3,6,9. But it is easy to check that the left-infinite periodic point ending with 2 differs only on the last letter of the left-infinite periodic point ending with 3, and similarly for 5,6 and 8,9. Hence there are really only 3 stepped line corresponding to left-infinite fixed points.

We can pair a right infinite and a left infinite periodic point to obtain a bi-infinite periodic point; but this makes sense from the viewpoint of symbolic dynamics only if the pair $a b$ at the origin of the periodic point occur in the language of the substitution, that is, in any sufficiently large image of a letter. An easy study shows that only 6 pairs can occur: $21,53,84$ and $61,93,34$.

If we draw the 6 stepped lines, the picture we obtain is striking (shown on Figure 9 for a large number of points). The three right infinite periodic points alternate with the 


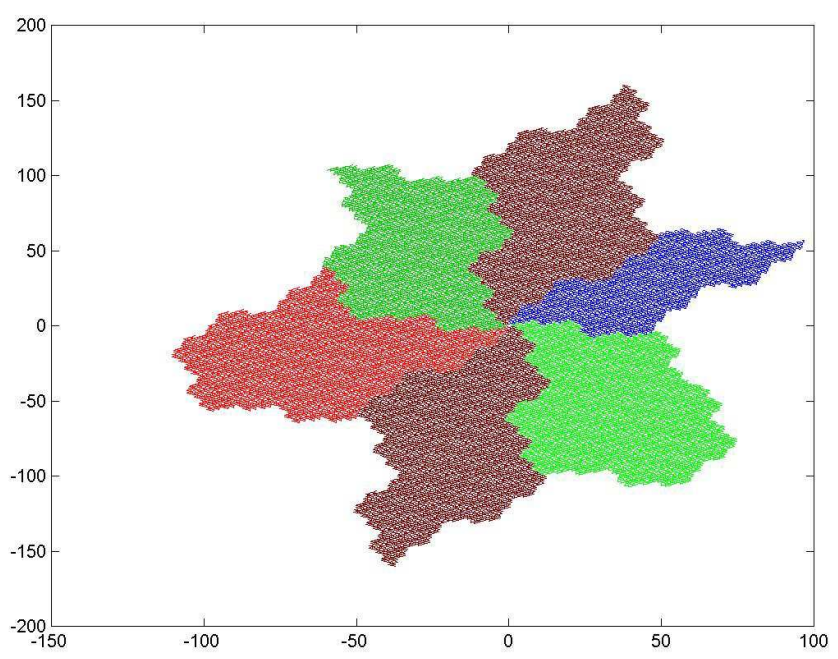

FiguRE 9. Long pieces of the stepped lines corresponding to the three positive fixed points and to the three negative fixed point.

three left infinite periodic points, each periodic point of one type being connected to the two neighbouring periodic points of the other type, and they seem to fill the whole plane. We are going to prove indeed that renormalizations of these stepped lines fill the plane.

Remark 37. The union of these 6 canonical lines is not exactly a cut and project scheme, but it is very close. If we fix one $K_{i}$ and we consider only the points of type a such that $\rho(a)=i$ then every point in the slice $x+\alpha y+\alpha^{2} z \in K_{i}-\alpha_{i}=\left[\beta_{i-1}-\alpha_{i}, \beta_{i}-\alpha_{i}\right]$ is obtained exactly once. Of course, points in the intersections of the slices can be obtained 2 or 3 times. Thus the union of lines appears as the superposition of three cut and projet schemes with different (but overlapping) windows. It might seem easier to restrict to one of the $K_{i}$ but self similarity would appear only by taking the cube of the induction. In some sense that is the strategy developed in [13] except that, since artificial importance is given to the point 0 , induction is done on a smaller interval and self-induction occurs only after the induction interval is indeed "inside" $K_{1}$.

6.2. Unstable plane in AntiTribonacci : renormalisation. We consider the family of sequences of finite stepped line defined, for all $a \in \mathcal{A}$, by

$$
\widetilde{\mathbf{L}}_{n}^{a}=M^{-n}\left(\mathbf{L}^{\sigma^{n}(a)}\right) \text {. }
$$

Using the results of Section 2.5, we see that the projections on the unstable space $\Pi_{\bar{T}, u}\left(\widetilde{\mathbf{L}}_{n}^{a}\right)$ of these sequences converge to a curve denoted $\mathcal{C}^{a}:\left[0 ; \Pi_{T, u}\left(e_{a}\right)\right] \rightarrow E_{\bar{T}, u}$. For all $n$ and all $0 \leq k \leq \sigma^{n}(a)$, if $t_{k}^{(n)}=\alpha^{n} \Pi_{T, u}\left(M^{-n} x_{k}^{\sigma^{n}(a)}\right)$, we have $\mathcal{C}^{a}\left(t_{k}^{(n)}\right)=\Pi_{\bar{T}, u}\left(M^{-n} x_{k}^{\sigma^{n}(a)}\right)$. We call elementary tiles the compact subsets $\mathcal{R}_{a}^{*}=\mathcal{C}^{a}\left(\left[0 ; \Pi_{T, u}\left(e_{a}\right)\right]\right)$.

Theorem 38. The compact sets $\left\{\mathcal{R}_{a}^{*}, a \in \mathcal{A}\right\}$ satisfy the system of set equation (5) below. As subset of the plane, each $\mathcal{R}_{a}^{*}$ has non empty interior.

Proof. First we make explicit the GIFS. Then, we prove that a particular union of translates of the tiles satisfies the same GIFS as the Tribonacci fractal. 


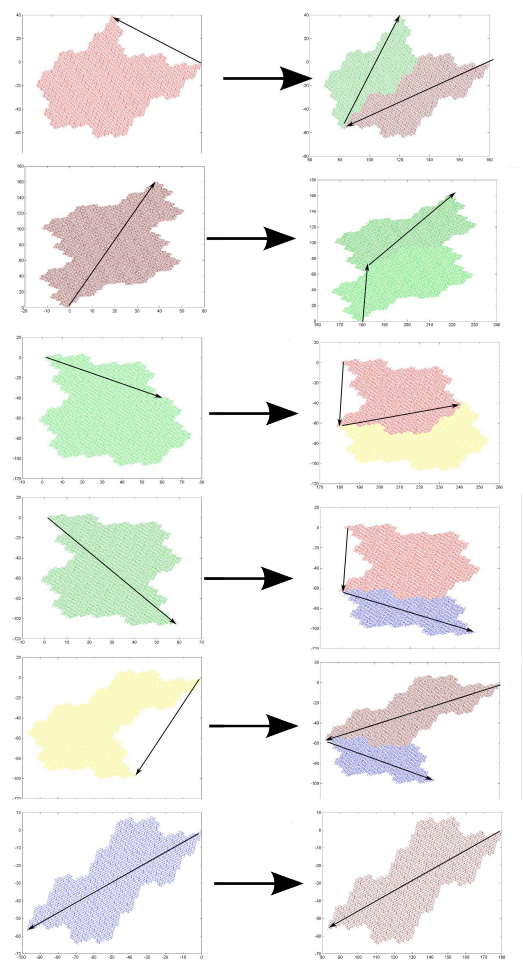

Figure 10. The IFS.

The GIFS is an immediate consequence of Proposition 21. There is a slight simplification: $\mathcal{R}_{2}^{*}=\mathcal{R}_{3}^{*}, \mathcal{R}_{5}^{*}=\mathcal{R}_{6}^{*}$ and $\mathcal{R}_{8}^{*}=\mathcal{R}_{9}^{*}$ since the corresponding projections are equal $\left(\Pi_{\bar{T}, u}\left(e_{2}\right)=\Pi_{\bar{T}, u}\left(e_{3}\right), \ldots\right)$, and the images are also equal modulo this identification. We obtain the system of set equation :

$$
\left\{\begin{array}{l}
\mathcal{R}_{1}^{*}=M^{-1} \mathcal{R}_{2}^{*} \cup\left(M^{-1} \Pi_{\bar{T}, u}\left(e_{2}\right)+M^{-1} \mathcal{R}_{5}^{*}\right) \\
\mathcal{R}_{2}^{*}=M^{-1} \mathcal{R}_{4}^{*} \cup\left(M^{-1} \Pi_{\bar{T}, u}\left(e_{4}\right)+M^{-1} \mathcal{R}_{5}^{*}\right) \\
\mathcal{R}_{4}^{*}=M^{-1} \mathcal{R}_{1}^{*} \cup\left(M^{-1} \Pi_{\bar{T}, u}\left(e_{1}\right)+M^{-1} \mathcal{R}_{7}^{*}\right) \\
\mathcal{R}_{5}^{*}=M^{-1} \mathcal{R}_{1}^{*} \cup\left(M^{-1} \Pi_{\bar{T}, u}\left(e_{1}\right)+M^{-1} \mathcal{R}_{8}^{*}\right) \\
\mathcal{R}_{7}^{*}=M^{-1} \mathcal{R}_{2}^{*} \cup\left(M^{-1} \Pi_{\bar{T}, u}\left(e_{2}\right)+M^{-1} \mathcal{R}_{8}^{*}\right) \\
\mathcal{R}_{8}^{*}=M^{-1} \mathcal{R}_{2}^{*}
\end{array} .\right.
$$

Let $W=825$. We claim that the union of tiles $\mathcal{R}_{W}^{*}=\mathcal{R}_{8}^{*} \cup\left(\Pi_{\bar{T}, u}\left(e_{8}\right)+\mathcal{R}_{2}^{*}\right) \cup\left(\Pi_{\bar{T}, u}\left(e_{8}+e_{2}\right)+\right.$ $\left.\mathcal{R}_{5}^{*}\right)$ is a Tribonacci fractal. We observe that $\sigma^{3}(W)=17183529352461845$. Identifying $2 \cong 3,5 \cong 6$ and $8 \cong 9$, we write $\hat{\sigma}^{3}(W)=\hat{\sigma}^{2}(25)(825) \hat{\sigma}(825) \hat{\sigma}^{2}(8)$.

Set $z=\Pi_{\bar{T}, u}\left(e_{\sigma^{2}(25)}\right)=\Pi_{\bar{T}, u}\left(e_{1718352}\right)$. Note that we have the equalities $\Pi_{\bar{T}, u}\left(e_{W}\right)=$ $\Pi_{\bar{T}, u}\left(e_{\sigma(W)}\right)=\Pi_{\bar{T}, u}\left(e_{\sigma^{2}(W)}\right)=0$, hence $\Pi_{\bar{T}, u}\left(e_{\sigma^{2}(25)}\right)+\Pi_{\bar{T}, u}\left(e_{\sigma^{2}(8)}\right)=0$. We obtain, using the cyclic decomposition of $\hat{\sigma}^{3}(W)$ : 

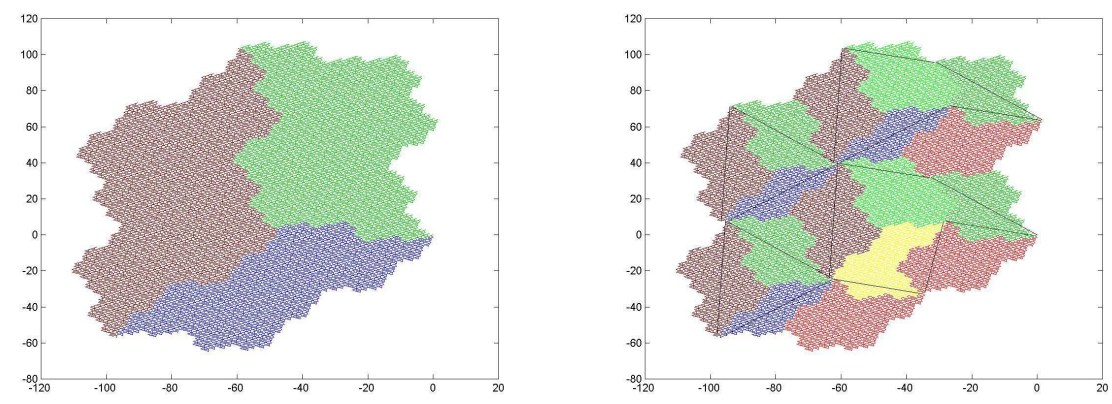

Figure 11. The set $\mathcal{R}_{W}^{*}$ decomposed in elementary tiles 825 and at level 3 in elementary tiles 17183529352461845 .

$$
\begin{gathered}
\Pi_{\bar{T}, u}\left(\mathcal{V}^{\sigma^{3}(W)}\right)=\Pi_{\bar{T}, u}\left(\mathcal{V}^{\sigma^{2}(25)}\right) \cup\left(z+\Pi_{\bar{T}, u}\left(\mathcal{V}^{W}\right)\right) \cup\left(z+\Pi_{\bar{T}, u}\left(\mathcal{V}^{\sigma(W)}\right)\right) \cup\left(z+\Pi_{\bar{T}, u}\left(\mathcal{V}^{\sigma^{2}(8)}\right)\right) \\
=\left(z+\Pi_{\bar{T}, u}\left(\mathcal{V}^{W}\right)\right) \cup\left(z+\Pi_{\bar{T}, u}\left(\mathcal{V}^{\sigma(W)}\right)\right) \cup\left(z+\Pi_{\bar{T}, u}\left(\mathcal{V}^{\sigma^{2}(8)}\right)\right) \\
\cup\left(z+\Pi_{\bar{T}, u}\left(e_{\sigma^{2}(8)}\right)+\Pi_{\bar{T}, u}\left(\mathcal{V}^{\sigma^{2}(25)}\right)\right) \\
=\left(z+\Pi_{\bar{T}, u}\left(\mathcal{V}^{W}\right)\right) \cup\left(z+\Pi_{\bar{T}, u}\left(\mathcal{V}^{\sigma(W)}\right)\right) \cup\left(z+\Pi_{\bar{T}, u}\left(\mathcal{V}^{\sigma^{2}(W)}\right)\right) .
\end{gathered}
$$

We deduce, applying $\sigma^{n}$ and $M^{-n}$, and letting $n \rightarrow \infty$, that

$$
\mathcal{R}_{W}^{*}=z+\left(M^{-3} \mathcal{R}_{W}^{*} \cup M^{-2} \mathcal{R}_{W}^{*} \cup M^{-1} \mathcal{R}_{W}^{*}\right)
$$

Recalling that the action of $M^{-1}$ on $E_{\bar{T}, u}$ is conjugate to a similarity of ratio $\mu$, as is the action of $M$ in the stable plane, we see that the unique solution of this set equation, up to a similitude, is the Tribonacci fractal. This result shows that at least one of the tiles $\mathcal{R}_{8}^{*}, \mathcal{R}_{2}^{*}$ or $\mathcal{R}_{5}^{*}$ has non empty interior. It then follows, by an obvious primitivity argument that all tiles have non empty interior.

Remark 39. The argument used in the proof shows that some pieces of the boundaries of the tiles coincide with pieces of boundaries of the Tribonacci fractal. A more careful combinatorial analysis would be required to understand exactly the structure of the boundary. But this gives some light to the result obtained in [13] about the dimension of the boundaries of the domains filled by the McMullen's curve.

Remark 40. Observe that the occurence of the Tribonacci fractal in this construction is somehow unexpected. We can not hope for such coincidence for general substitutions since the action of $M$ on the contracting space has no reason to be conjugate to the action of $M^{-1}$ on the expanding space. A necessary condition is the reciprocity of the characteristic polynomial. This is true for geometric reasons in the case of self-induced IET coded by continuity intervals. We do not know whether it is always sufficient in the general case.

\subsection{McMullen's discrete curve.}

Definition 41. We denote by $Q$ the bijection from $\mathbb{Z}^{3}$ onto $\frac{1}{2} \mathbb{Z}[\alpha]$ defined by:

$$
Q(n, p, q)=\frac{n}{2}+\frac{p}{2} \alpha+\frac{q}{2} \alpha^{2} .
$$


The set $\mathcal{Z}=Q^{-1}\left(\frac{1}{2} \mathbb{Z}[\alpha] \cap\left[\beta_{0}, \beta_{3}[)\right.\right.$ is a "slice" in $\mathbb{Z}^{3}$ between two planes. It is partitioned in two cosets, depending on whether the parity of $n+p+q$ is odd or even, which are exchanged by the involution $I_{\left[\beta_{0}, \beta_{3}[\right.}: x \mapsto x+1 / 2 \bmod 1$ on the interval. Each coset is in correspondence with $\mathbb{Z}^{2}$ by the following trivial lemma :

Lemma 42. The map $\mathcal{Z} \rightarrow \mathbb{Z}^{2}, \frac{n}{2}+\frac{p}{2} \alpha+\frac{q}{2} \alpha^{2} \mapsto(p, q)$ is two-to-one, since for all $(p, q) \in \mathbb{Z}^{2}$ there is a unique $n$ such that $Q(n, p, q) \in\left[\beta_{0}, \beta_{3}\left[, Q(n+1, p, q) \in\left[\beta_{0}, \beta_{3}[\right.\right.\right.$, i.e. exactly two points in the slice. It is one-to-one on each coset of a given parity.

Definition 43. We call McMullen's curve and we denote by $\mathcal{L}$ the stepped line which admits as ordered sequence of vertices the lift to $\mathbb{Z}^{3}$ of the orbit of the fixed point $\alpha_{1}$, $\left(Q^{-1}\left(T^{n}\left(\alpha_{1}\right)\right)\right)_{n \geq 0}$.

It is a stepped line since the differences $Q^{-1}\left(T^{n+1}\left(\alpha_{1}\right)\right)-Q^{-1}\left(T^{n}\left(\alpha_{1}\right)\right)$ are given by $Q^{-1}\left(t_{u_{n}}\right)$, and it is the image of the stepped line $\mathbf{L}^{u}$ by the map $e_{a} \mapsto Q^{-1}\left(t_{a}\right)$, for $a \in \mathcal{A}$.

This stepped line is a piece of a more complete object: a family of stepped lines that partition the slice $\mathcal{Z}$. Let $z$ be any point in $\mathcal{Z}$. Since $Q(z) \in\left[\beta_{0}, \beta_{3}[\right.$ we can set $\mathcal{T}(z)=$ $Q^{-1}(T(Q(z)))$. This defines a map from $\mathcal{Z}$ to itself, and by construction $\mathcal{T}(z)-z$ take values in the finite set $\left\{Q^{-1}\left(t_{a}\right), a \in \mathcal{A}\right\}$. Hence through any point $z_{0}$ in $\mathcal{Z}$ goes a biinfinite stepped line $\left(z_{n}\right)_{n \in \mathbb{Z}}$ connecting $z_{n}$ to $z_{n+1}=\mathcal{T}\left(z_{n}\right)$. The sets of vertices of these stepped lines are infinite since, by minimality, the IET has no periodic points. This family of curves is well understood:

Proposition 44. Each of these stepped lines is contained in a coset of fixed parity. The odd coset is the disjoint union of the three stepped lines through the points $Q^{-1}\left(\alpha_{1}\right)$, $Q^{-1}\left(\alpha_{2}\right), Q^{-1}\left(\alpha_{3}\right)$; the stepped line $\mathcal{L}$ is the "positive half" of one of those, namely the one starting at $Q^{-1}\left(\alpha_{1}\right)$. The even coset is the disjoint union of the image of these three stepped lines by the involution $I_{\left[\beta_{0}, \beta_{3}[\text {. }\right.}$.

Proof. Parity is preserved along a stepped line, since elementary moves do not change it. Hence each stepped line is contained in one coset. The projection on the last two coordinates then becomes one-to-one, and we obtain a picture in $\mathbb{Z}^{2}$.

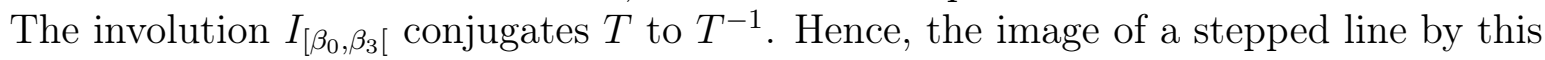
involution is a stepped line in the other coset, which projects to the same stepped line in $\mathbb{Z}^{2}$,with opposite orientation.

We claim that there are exactly six distinct bi-infinite stepped lines (three in the odd coset and three in the even coset) covering all $\mathcal{Z}$. Figure 12 shows how the three even stepped lines behave close to the origin. Figure 13 shows the "positive" halves of the same three stepped lines seen at a larger scale. Let us give an idea of the argument, similar to the one developed in [13]. Consider a point $z_{0} \in \mathcal{Z}$ which is in the odd coset, and denote $L_{0}$ the stepped line going through $z_{0}$. Since the return time to any interval of positive measure is bounded, there is a constant $C$ (independent of $z_{0}$ ) such that there is a point $z_{0}^{\prime} \in L_{0}$ with $Q\left(z_{0}^{\prime}\right) \in J_{a}$ and $\left\|z_{0}-z_{0}^{\prime}\right\|<C$. Denote $z_{1}=M^{-1} z_{0}^{\prime}$. Since $Q\left(z_{0}^{\prime}\right) \in J_{a}$, $z_{1} \in \mathcal{Z}$. Moreover $\left\|z_{1}\right\| \leq|\mu|\left(\left\|z_{0}\right\|+C\right)$. There is a constant $B(B=2 C /(1-|\mu|))$ such that, iterating this procedure, we end up (after finite time for all initial $z_{0}$ ) in the ball of radius $B$. A direct computation shows that the intersection of the odd coset with the ball of radius $B$ contains only stepped lines issued from a fixed point. Using the self similarity of these lines, we can go back to $z_{0}$ to deduce that it was in such a stepped line. Hence the 
odd coset is partitioned by the stepped lines corresponding to the three periodic points, and the even coset by their images by the involution.

It is delicate (but done in [13]) to prove that there are no crossings (neither self crossings, neither crossings between two of the three stepped lines of a class) in the planar projections (on $\mathbb{Z}^{2}$ and on the "stable" plane). A combinatorial analysis of the finite number of possibilities provides a proof. It follows from this last statements that a renormalisation of the three stepped lines of a class must fill the plane with three (essentially disjoint) subsets. We will be able to give a precise description of the boundaries of these domains (there was an attempt in [13]).

Since ${ }^{t} A^{-1}$ is the companion matrix of the minimal polynomial of $\alpha$, we have $\alpha Q(v)=$ $Q\left({ }^{t} A^{-1} v\right)$. Hence multiplication by $\alpha$ seen in $\mathcal{Z}$ splits into a contraction in the direction $\left(1, \alpha, \alpha^{2}\right)$ and an expansion (conjugate to a similarity of ratio $\beta$ ) in the plane $x+\alpha y+\alpha^{2} z=$ 0 . We will see that it is rather natural to project the McMullen's stepped lines on this plane.

Remark 45. We propose still a more general construction, similar to what is done in [13]. Starting with a given point in $x \in\left[\beta_{0}, \beta_{3}\right)$ we consider in $\mathbb{Z}^{3}$ the bi-infinite stepped line defined by $z_{0}=0$ and, for all $n \in \mathbb{Z}, z_{n+1}-z_{n}=Q^{-1}\left(T^{n+1}(x)\right)-Q^{-1}\left(T^{n}(x)\right)$. We claim that, generically, the projection of this stepped line onto $\mathbb{Z}^{2}$ fills $\mathbb{Z}^{2}$; for a countable set of points (the orbit of the discontinuities) there are three stepped lines, and for an uncountable set of Lebesgue measure 0, there are two stepped lines.

This is reminiscent of the structure of the space of Penrose tilings, where some special tilings have symmetry of order 2 or 10, and generic tilings do not have these symmetries.

We now prove that McMullen's curve $\mathcal{L}$ can be interpreted as yet another projection (up to a translation) of the stepped line $\mathbf{L}^{u}$. It will be convenient to generalize the map $Q$ to any three dimensional lattice with a fixed basis.

Definition 46. Let $E$ be a 3-dimensional vector space with a basis $\mathcal{B}$. We define the map $Q_{E, \mathcal{B}}$, from the lattice generated by $\mathcal{B}$ to $\frac{1}{2} \mathbb{Z}[\alpha]$, by $Q_{E, \mathcal{B}}(v)=\frac{n}{2}+\frac{p}{2} \alpha+\frac{q}{2} \alpha^{2}$ if $v$ has coordinates $(n, p, q)$ in the basis $\mathcal{B}$.

We use the basis $\hat{\mathcal{B}}$ defined in Section 9.2 of Appendix, and the projection $\hat{\Pi}$ to $E_{\bar{T}}$ defined in that section by $\hat{\Pi}=\Pi_{F} \circ\left(\Pi_{j} \oplus \Pi_{\bar{T}}\right)$, where $\Pi_{F}$ keeps invariant the basis $\left\{\hat{e}_{4}, \hat{e}_{5}, \hat{e}_{6}\right\}$ of $E_{\bar{T}}$, sends $E_{T}$ to 0 , and sends $\hat{e}_{i}$ to $\hat{e}_{i+3}$ for $i=1,2,3$. We see this projection $\hat{\Pi}$ as a map from $\mathbb{R}^{\mathcal{A}}$, endowed with the canonical basis, to $E_{\bar{T}}$, endowed with the basis $\left\{\hat{e}_{4}, \hat{e}_{5}, \hat{e}_{6}\right\} \subset \hat{\mathcal{B}}$, and we identify in this way $E_{\bar{T}}$ and $\mathbb{R}^{3}$.

Theorem 47. We have:

$$
\hat{\Pi}\left(\boldsymbol{L}^{u}\right)=\mathcal{L}-Q^{-1}\left(\alpha_{1}\right)
$$

\section{Proof.}

This equality makes sense since $\hat{\Pi}\left(\mathbf{L}^{u}\right)$ is contained in $E_{\bar{T}}$ and $\mathcal{L}$ is included in $\mathbb{R}^{3}$, and we have identified these two spaces.

It is a direct consequence of Lemma 57 (in Appendix). Indeed, by definition, successive points in $\hat{\Pi}\left(\mathbf{L}^{u}\right)$ satisfy:

$$
\hat{\Pi}\left(x_{n+1}^{u}\right)=\underset{30}{\hat{\Pi}}\left(x_{n}^{u}\right)+\hat{\Pi}\left(e_{u_{n}}\right)
$$




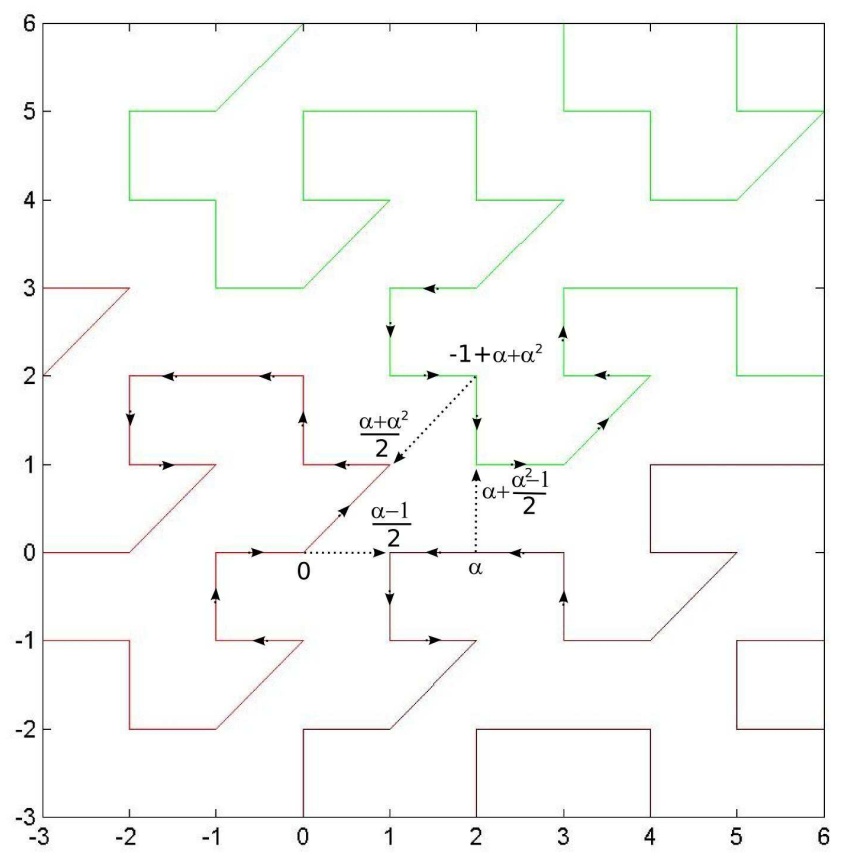

Figure 12. Projection on $\mathbb{Z}^{2}$ of three stepped lines corresponding to forward orbits of $0, \alpha$ and $-\alpha^{3}$. Dotted arrows correspond to the other possibilities at the singularities (if the intervals were left open instead of being left closed in the definition of the IET. Points represented correspond to points $(n, p, q) \in \mathcal{Z}$ with $n+p+q$ even.

while successive points in $\mathcal{L}$ satisfy:

$$
Q^{-1}\left(T^{n+1}\left(\alpha_{1}\right)\right)=Q^{-1}\left(T^{n}\left(\alpha_{1}\right)\right)+Q^{-1}\left(t_{u_{n}}\right) .
$$

But Lemma 57 asserts that $\hat{\Pi}\left(e_{a}\right)=Q_{E_{\bar{T}}, \hat{\mathcal{B}_{\bar{T}}}}^{-1}\left(t_{a}\right)$, hence the two lines have same increments. The theorem follows immediately from the fact that the line $\hat{\Pi}\left(\mathbf{L}^{u}\right)$, by definition, begins at the origin, while $\mathcal{L}$ begins at $Q^{-1}\left(\alpha_{1}\right)$.

It happens that this stepped line is very close to the projection $\Pi_{\bar{T}}$ of the stepped line $\mathbf{L}^{u}$ on the space $E_{\bar{T}}$; the difference being related to what happens in the "neutral" direction. To make this dependence more explicit, let us now define a piecewise translation from $\mathbb{Z}^{3}$ (as the space of the McMullen's curve) to $\mathbb{Z}^{3}$ (as the space $E_{\bar{T}}$ ), by

$$
K(z)=z-\sum_{i=1}^{3} Q^{-1}\left(\alpha_{i}\right) \mathbf{1}_{K_{i}}(Q(z)) .
$$

Observe that $K(\mathcal{L})$ is also a stepped line since the number of possible translations is finite and indeed,

\section{Theorem 48.}

$$
\Pi_{\bar{T}}\left(\boldsymbol{L}^{u}\right)=K(\mathcal{L}) .
$$




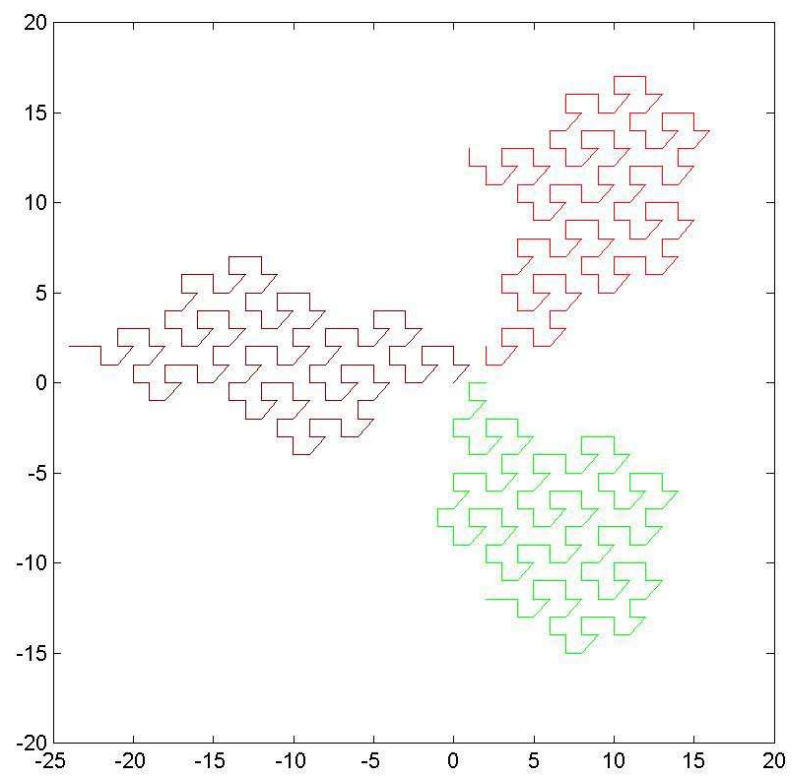

FiguRE 13. McMullen's curves corresponding to forward orbits of the three fixed points. Projection on $\mathbb{Z}^{2}$.

Proof. We observe that for all $i$ and all $z \in \mathcal{Z}$ with $Q(z) \in K_{i}, Q(K(z))=Q(z)-\alpha_{i}$. It follows from Theorem 47 and from the proof of Theorem 31 that

$$
T^{n}\left(\alpha_{1}\right)=\sum_{i=1}^{n} Q\left(\Pi_{\bar{T}}\left(e_{u_{i}}\right)+\Pi_{j}\left(e_{u_{i}}\right)\right)=Q\left(\Pi_{\bar{T}}\left(x_{n}^{u}\right)\right)-\alpha_{k^{-}\left(u_{n}\right)} .
$$

This result shows that it is possible to switch from $\mathbf{L}^{u}$ to $\mathcal{L}$ by moving points by (uniformly bounded) translations. It is worth observing that, by definition, the McMullen's curve has no multiple points. Hence these "translations" do split the multiple points of $\mathbf{L}^{u}$. Of course the advantage of $\mathbf{L}^{u}$ is that its self-similarity properties are much simpler than those of the McMullen's curve. As we will see in the next section, since the two curves remain at bounded distance, the limiting Peano curves are the same.

6.4. Renormalized McMullen curve. We define the concatenation of two Peano limit curves $\mathcal{C}^{a}$ and $\mathcal{C}^{b}$ (defined as in Section 6.2) as the Peano limit curve obtained from $a b$ denoted $\mathcal{C}^{a b}$. We naturally extend this notation to unbounded curves associated with infinite words.

The stepped line $\mathcal{L}$ is somehow self-similar. But the self-similarity at finite scales is not completely obvious and the easiest way to analyse the limiting rescaled curve is to compare it with $\Pi_{\bar{T}, u}(\mathcal{V})$. Assume we have a parametrization of $\mathcal{L}$, that is a bijective map $\varphi$ from $\mathbb{R}^{+}$onto the union of the segments joining successive points of $\mathcal{L}$. Define the map $\Pi_{\alpha}$ as the projection on the unstable plane of the matrix $A$ along its stable line. We call 
renormalized McMullen curve the limiting curve:

$$
\widetilde{\mathcal{L}}(t)=\lim _{n \rightarrow \infty} \Pi_{\alpha}\left({ }^{t} A^{-3 n} \varphi\left(\alpha^{-3 n} t\right)\right) .
$$

This limiting curve is well defined, and is exactly $\mathcal{C}^{u}$.

Theorem 49. The renormalized projection of the McMullen curve tends to a space filling curve $\widetilde{\mathcal{L}}$ which is equal to the Peano curve $\mathcal{C}^{u}$.

Proof. Formally, this result is a consequence of Theorem 48; indeed, since the two stepped lines remain at uniformly bounded distance, their renormalisation by similarity must have the same limit. A natural parameterization for the McMullen's curve is obtained by taking the natural one for the projection $\mathcal{L}=\hat{\Pi}\left(\mathbf{L}^{u}\right)$ giving a length $\Pi_{\bar{T}, u}\left(e_{a}\right)$ to a step in direction $e_{a}$.

The idea behind this proof is that what happens in the neutral direction vanishes in the limit because of the contraction. But at finite stages the identifications $2 \cong 3,6 \cong 7$ and $8 \cong 9$ do not hold for the McMullen's curve, so a complete formalization would be heavy, as well as a direct study of the McMullen's curve. In other words, the projection of McMullen's curve on the plane $E_{\bar{T}, u}$ is obtained by a projection $\Pi_{\bar{T}, u} \circ \hat{\Pi}$ which does not commute with $M$, but such that the conjugates $M^{-n} \Pi_{\bar{T}, u} \hat{\Pi} M^{n}$ converge to the projection $\Pi_{\bar{T}, u}$.

Remark 50. The union of the renormalizations of the three McMullen's curves fills the plane and a more specific analysis of their shape follows from analysis of the space filling curves $\mathcal{C}^{a}$.

\section{DUAL}

7.1. Dual substitution. We consider the following substitution, called dual substitution of $\sigma$,

$$
\begin{aligned}
\sigma_{*}: & 1 \rightarrow 456 \\
2 & \rightarrow 78 \\
3 & \rightarrow 91 \\
4 & \rightarrow 23 \\
5 & \rightarrow 12 \\
6 & \rightarrow 3 \\
7 & \rightarrow 4 \\
8 & \rightarrow 5 \\
9 & \rightarrow 67 .
\end{aligned}
$$

Its matrix is the transpose ${ }^{t} M$ of $M$. The choice of the ordering is given by the way the continuity intervals of $T$ are ordered on the interval. Indeed, this substitution describes the way the dynamical partition splits after induction. We stress again that there is no clear definition of such a "dual" substitution without the help of the geometry of the underlying IET.

We decompose $\mathbb{R}^{\mathcal{A}}$ into 3 stable subspaces, $E_{n}^{*}, E_{T}^{*}$ and $E_{\bar{T}}^{*}$ (with the natural notations for the corresponding projections). The relation $M=P^{-1} B P$ transposes into ${ }^{t} M=$ ${ }^{t} P^{t} B^{t} P^{-1}$. Hence basis vectors for the stable spaces can be taken to transposes of row vectors of $P^{-1}$. We observe that $\sigma_{*}$ has six periodic points (of period 3 ), respectively 
limits of $\sigma_{*}^{3 n}(4), \sigma_{*}^{3 n+1}(4)=\sigma_{*}^{3 n}(2), \sigma_{*}^{3 n+2}(4)=\sigma_{*}^{3 n}(7)$ and $\sigma_{*}^{3 n}(3), \sigma_{*}^{3 n+1}(3)=\sigma_{*}^{3 n}(9)$, $\sigma_{*}^{3 n+2}(3)=\sigma_{*}^{3 n}(6)$.

We could do various things to study the stepped line(s) associated with this substitution. But we decided to focus on its projection on the unstable space $E_{\bar{T}}^{*}$ because we recover the Peano curve obtained by Arnoux in [1] (and by ourselves in Section 4.5) as limit Peano curve of a sequence of renormalized stepped lines. This indeed provides an IFS construction of the curve and this question has been one of the starting points of this work.

7.2. Unstable space in dual AntiTribonacci. The space $E_{\bar{T}, u}^{*}$ is the unstable space associated with the eigenvalues $\beta$ and $\bar{\beta}$ of the matrix ${ }^{t} M$. In this subspace, it makes sense to use the theory developed in Section 2.5 to investigate the stepped lines $\Pi_{\bar{T}, u}^{*}\left(M^{-N}\left(\mathbf{L}_{*}^{\sigma_{*}^{N}(a)}\right)\right)$ for $a \in \mathcal{A}$ and their limiting curves $\mathcal{C}_{*}^{a}$. To illustrate the link with the work of Arnoux [1], we want to show how the circle $\mathbb{S}^{1}$ can be naturally embedded in a Tribonacci Fractal using these limiting curves. For all $a \in \mathcal{A}$ we can see $\mathcal{C}_{*}^{a}$ as a map defined on $I_{a}$. Concatenation of the stepped lines (as in Section 6.4) in the order 789123456 would yield a map defined on $\mathcal{I}$ and our point is that its image is a Tribonacci fractal. For technical reasons it is more convenient to state the result using the order prescribed by the image partition $T(\mathcal{I})$ and to move the origin ; we consider the concatenation of the curves in the order $W=341267598$. In this setting, the fixed points are at boundaries of the intervals and they are mapped on the origin. If we define $e_{a}=-\Pi_{\bar{T}, u}^{*}\left(e_{7}\right), e_{b}=\Pi_{\bar{T}, u}^{*}\left(e_{1}\right)$, $e_{c}=-\Pi_{\bar{T}, u}^{*}\left(e_{4}\right)$, we obtain a basis of the 3 -dimensional space $E_{\bar{T}, u}^{*}$, which can be seen as $\mathbb{R}^{\breve{\mathcal{A}}}$ on which ${ }^{t} M^{-1}$ acts as $A$; the Tribonacci fractal $\breve{\mathcal{R}}$ can be embedded in this way in $E_{\bar{T}, u}^{*}$.

Theorem 51. In $E_{\bar{T}, u}^{*}$,

$$
\mathcal{R}_{W}^{*}:=\lim _{N \rightarrow \infty}\left(\Pi_{\bar{T}, u}^{*}\left({ }^{t} M^{-N} \mathcal{V}^{\sigma_{*}^{N}(W)}\right)\right)=\breve{\mathcal{R}} .
$$

The limiting curve is a space-filling curve filling precisely the Tribonacci Rauzy fractal. This result could be proved using the GIFS strategy already developped, for instance showing that it satisfies the characteristic system of set equations of the Tribonacci Rauzy fractal. We adopt here a different strategy in order to illustrate the relation between the dual substitution and the substitution itself : we want to show that the "contraction" procedure for $\sigma$ and the "expanding" procedure for $\sigma_{*}$ both yield representations of $\mathbb{S}^{1}$ (in fact of three pieces of the stable manifold of the pseudo-Anosov underlying the induction).

Proof. We consider a point $x \in \mathbb{S}^{1}$. We propose to describe it in two ways: on the one hand, by following its symbolic dynamics (driven by substitution $\sigma$ ); on the other hand, by computing, at each level of refinement of the dynamical partition (driven by $\sigma_{*}$ ) the interval to which it belongs. These two symbolic descriptions yield two infinite sequences of prefixes for $\sigma$ and $\sigma_{*}$ (which happen to be paths with opposite orientation in the same graph by a general argument). The "contracting" construction (Section 2.4) for the substitution $\sigma$ and the "expanding" construction (Section 2.5) for the dual substitution $\sigma_{*}$ yield two maps from $\mathbb{S}^{1}$ to $\mathbb{R}^{3}$. The amazing fact is that these maps are the same (provided the basis are chosen correctly). Observe that there are two dualities involved here. Indeed, we already noticed that the "contracting" and "expanding" procedures 
yield dual GIFS (GIFS with transposed matrices) while we apply those dual procedures respectively to $\sigma$ and to its dual $\sigma_{*}$.

Let us be more specific. Let $x \in \mathbb{S}^{1}$ and set $v=\varphi_{\mathcal{I}}(x)$. It is standard to associate to $x$ an infinite path in the prefix automaton of $\sigma$. Here, this path is determined by the sequence of vertices $\left(a_{n}\right)_{n \geq 0}$ and related to the symbolic dynamics as follows : for all $n \geq 0, \sigma\left(a_{n+1}\right)=P_{n} a_{n} S_{n}$ while $v=\lim _{n \rightarrow \infty} S_{0} \sigma\left(S_{1}\right) \cdots \sigma^{n}\left(S_{n}\right)$. Observe $\sigma^{n}\left(a_{n}\right)=\sigma^{n-1}\left(P_{n-1}\right) \cdots \sigma\left(P_{1}\right) P_{0} a_{0} S_{0} \sigma\left(S_{1}\right) \cdots \sigma^{n-1}\left(S_{n-1}\right)$. We consider the sequence of labels $\left(P_{n}\right)_{n \geq 0}$.

We observe that $a_{0}$ is the index of the interval of the partition $T(\mathcal{I})$ in which lies $x$. Let us consider the (unique) prefix $P_{a_{0}}^{W}$ of the word $W=341267598$ such that $P_{a_{0}}^{W} a_{0} S_{a_{0}}^{W}=W$ for some suffix $S_{a_{0}}^{W}$ (that is the list of the names of the intervals of $T(\mathcal{I})$ lying between $\alpha_{3}$ and the interval containing $x$ ). The induction yields successive refinement of the partition $T(\mathcal{I})$ made of intervals of the form $T^{k}\left(h^{n}\left(I_{b}\right)\right.$ ) (at level $n$, for $k$ integers and $b \in \mathcal{A}$ ), called intervals of type $b$. At each level, an interval of type $a$ is tiled by intervals of the next level, of types given (in the right order) by $\sigma_{*}(a)$ (by construction of $\sigma_{*}$ ). We observe that $a_{n}$ is the type of the interval to which $x$ belongs at level $n$. Since each letter appears at most once in each image, we can decompose $\sigma_{*}\left(a_{n-1}\right)=P_{n-1}^{*} a_{n} S_{n-1}^{*}$. It determines a sequence $\left(P_{n}^{*}\right)_{n \geq 0}$ of prefixes which is the sequence of labels of a backward path in the prefix automaton of $\sigma_{*}$ (and indeed of the same path seen in backward direction). The position of $x$ in the partition $T(\mathcal{I})$ is determined by the sequence $P_{a_{0}}^{W}, P_{0}^{*}, \ldots, P_{n}^{*}, \ldots$ in the sense that at level $n$ the word $\sigma_{*}^{n}(W)$ gives the list of the types of the intervals of level $n$ tiling $\mathbb{S}^{1}$ (starting at $\left.\alpha_{3}\right)$ and the decomposition $\sigma_{*}^{n}(W)=\sigma_{*}^{n}\left(P_{a_{0}}^{W}\right) \sigma_{*}^{n-1}\left(P_{0}^{*}\right) \cdots P_{n-1}^{*} a_{n} S_{n-1}^{*} \cdots \sigma_{*}^{n-1}\left(S_{0}^{*}\right) \sigma_{*}^{n}\left(S_{a_{0}}^{W}\right)$ specifies the position of the copy of $h^{n}\left(I_{a_{n}}\right)$ containing $x$.

We are going to use these two sequences $\left(P_{n}\right)_{n \geq 0}$ and $\left(P_{n}^{*}\right)_{n \geq 0}$ of prefixes to build two natural maps valued in $E_{T, s}$ and in $E_{\bar{T}, u}^{*}$ respectively.

On the one hand, using the sequence $\left(P_{n}\right)_{n \geq 0}$ we define a map on $\mathbb{S}^{1}$ following the "contracting" construction proposed in Section 2.4. Consider the projections on $E_{T, s}$ of the (finite) stepped lines associated with $\sigma^{n}\left(P_{n}\right) \cdots \sigma\left(P_{1}\right) P_{0} a_{0}$. By the same arguments as those of Proposition 13, this sequence converges. We set

$$
Y(x)=\Pi_{T, s} e_{a_{0}}+\sum_{i \geq 0} M^{i} \cdot \Pi_{T, s} e_{P_{i}} .
$$

On the other hand, following the procedure developped in Section 2.5, we associate to the sequence $\left(P_{n}^{*}\right)_{n \geq 0}$ a point in the limiting curve $\mathcal{C}_{*}^{W}$ by

$$
Y^{*}(x)=\Pi_{\bar{T}, u}^{*} e_{P_{a_{0}}^{W}}+\sum_{n \geq 0}{ }^{t} M^{-n-1} \Pi_{\bar{T}, u}^{*} e_{P_{n}^{*}} .
$$

Let us identify $E_{T}$ and $E_{\bar{T}}^{*}$ with $\mathbb{R}^{\breve{\mathcal{A}}}$ by the maps,

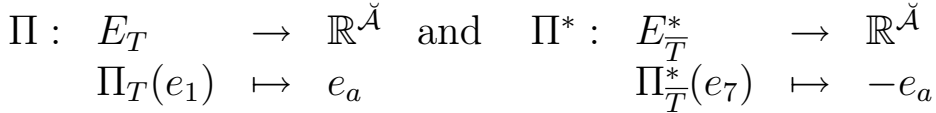

$$
\begin{aligned}
& \Pi_{T}\left(e_{5}\right) \mapsto e_{b} \quad \Pi_{T}^{*}\left(e_{1}\right) \mapsto e_{b} \\
& \Pi_{T}\left(e_{8}\right) \mapsto e_{c} \quad \Pi_{\bar{T}}^{*}\left(e_{4}\right) \mapsto-e_{c} .
\end{aligned}
$$

We observe that in the basis of $E_{T}$ (resp. of $E_{\bar{T}}^{*}$ ), the matrix $M$ (resp. ${ }^{t} M^{-1}$ ) acts as the Tribonacci matrix, i.e $\Pi M=A \Pi$ (resp. $\left.\Pi^{* t} M^{-1}=A \Pi^{*}\right)$. We claim that, 


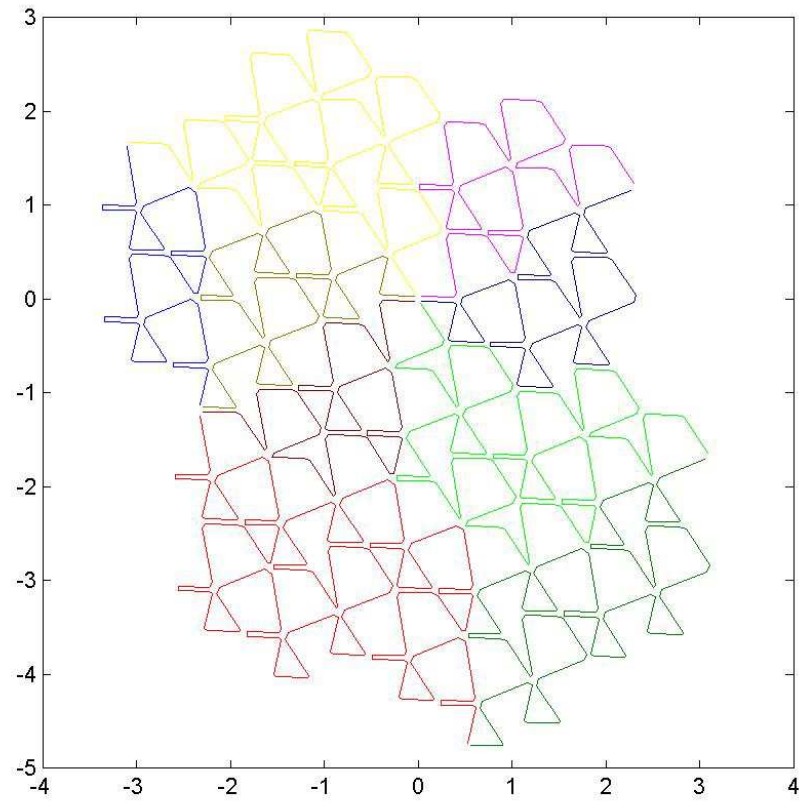

FIGURE 14. A view of $\Pi_{\bar{T}, u}^{*}\left(M^{-6} \mathcal{V}^{\sigma^{6}(W)}\right)$ with colors corresponding to images of the different letters. Multiple points have been artificially split to allow to "follow" the curve.

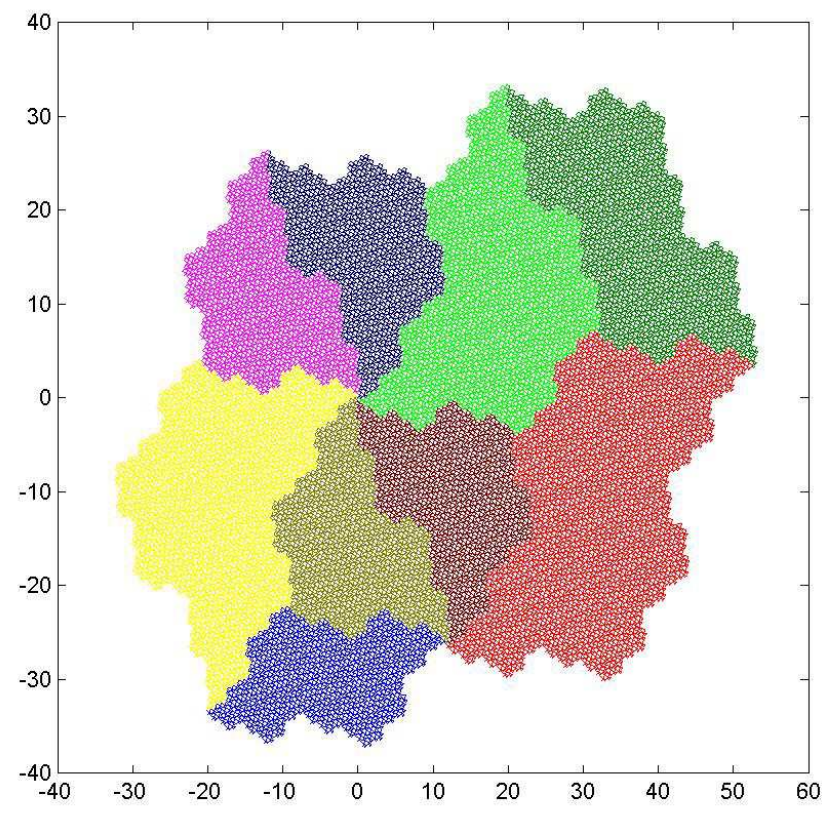

FIGURE 15. The limiting space filling curve $\lim _{N \rightarrow \infty} \Pi_{\bar{T}, u}^{*}\left(M^{-N} \mathcal{V}^{\sigma^{N}(W)}\right)$ 
Proposition 52. For all $x \in \mathbb{S}^{1}$,

$$
\Pi(Y(x))=\Pi^{*}\left(Y^{*}(x)\right) .
$$

We denote $\breve{\Pi}_{s}$ the projection of $\mathbb{R}^{\breve{A}}$ on the stable plane of $A$ parallel to the expanding direction. We observe that $\breve{\Pi}_{s} \Pi=\Pi_{T, s}$ on $E_{T}$ and that $\breve{\Pi}_{s} \Pi^{*}=\Pi^{*} \Pi_{T, u}^{*}$ on $E_{\bar{T}}^{*}$. Recalling that $\Pi_{T, s} e_{i}=\Pi_{T, s} e_{j}$ as soon as $\rho(i)=\rho(j)$ we obtain, as in Theorem 26,

$$
\Pi Y(x)=\breve{\Pi}_{s} e_{\rho\left(a_{0}\right)}+\sum_{i=0}^{\infty} A^{i} \breve{\Pi}_{s} e_{\rho\left(P_{i}\right)} .
$$

We denote for all $a \in \mathcal{A}, w_{a}=\Pi_{\bar{T}}^{*}\left(e_{a}\right)$ and for all $V=v_{1} \cdots v_{n} \in \mathcal{A}^{*}, w_{V}=\sum_{i=1}^{n} w_{v_{i}}$; with this notation,

$$
\Pi^{*} Y^{*}(x)=\breve{\Pi}_{s}\left(w_{P_{a_{0}}^{W}}+\sum_{i=0}^{N-1} A^{i+1} w_{P_{i}^{*}}\right) .
$$

The final result relies on the following technical computation :

Lemma 53.

$$
A w_{P_{0}^{*}}+w_{P_{a_{0}}^{W}}-A w_{P_{a_{1}}^{W}}=e_{\rho\left(P_{0}\right)}+e_{\rho\left(a_{0}\right)}-A e_{\rho\left(a_{1}\right)} .
$$

The proof consists in checking a finite number (16) of identities and is given in Section 9.3. We stress that it is purely computational and that we have still no (convincing) conceptual argument to guarantee such coincidence in a more general context. Now, we use Lemma 53 inductively to show that, for all $N$,

$$
w_{P_{a_{0}}}+\sum_{i=0}^{N-1} A^{i+1} w_{P_{i}^{*}}=e_{\rho\left(a_{0}\right)}+\sum_{i=0}^{N-1} A^{i} e_{\rho\left(P_{i}\right)}+A^{N}\left(w_{P_{a_{N}}^{W}}\right)-A^{N}\left(e_{\rho\left(a_{N}\right)}\right) .
$$

We observe that $\breve{\Pi}_{s} A^{N}\left(w_{P_{a_{N}}^{*}}\right) \rightarrow 0$ and $\breve{\Pi}_{s} A^{N}\left(e_{\rho\left(a_{N}\right)}\right) \rightarrow 0$. Hence, taking the projection and letting $N$ tend to infinity, we conclude

$$
\Pi^{*} Y^{*}(x)=\Pi^{*}\left(\Pi_{\bar{T}, u}^{*} e_{P_{a_{0}}^{W}}+\sum_{i=0}^{\infty}{ }^{t} M^{-i-1} \Pi_{\bar{T}, u}^{*} e_{P_{i}^{*}}\right)=\breve{\Pi}_{s} e_{\rho\left(a_{0}\right)}+\sum_{i=0}^{\infty} A^{i} \breve{\Pi}_{s} e_{\rho\left(P_{i}\right)} .
$$

So the claim is proved. We already know (by simple adaptation of Corollary 27 ) that the image of $\Pi \circ Y$ is the Tribonacci fractal ; hence, since $\mathcal{R}_{W}^{*}=\Pi^{*} Y^{*}\left(\mathbb{S}^{1}\right)$, the theorem is proved as well.

\section{Additional REMarks}

8.1. Surfaces diffeomorphisms. We have not considered here the pseudo-Anosov map which is associated with this interval exchange transformation. Let us just remark here that the points $\alpha_{i}$ appear naturally in this setting as singular points on the surface, and the intervals $K_{i}$ as initial segments of separatrices at the singularity. The pseudo-Anosov preserves the singularity, but exchanges the separatrices. This helps to explain why it is reasonable to consider the interval exchange as defined, not on a circle, but on the union of three segments; it is just by coincidence that the endpoints of these segments turn out to be on the same orbit of the vertical flow, so that we can identify the extremities of the segments by sliding them along vertical orbits, and obtain a circle transverse to the vertical flow. 
The interval exchange is measurably isomorphic to the substitution dynamical system associated with the Tribonacci substitution; in this respect, Tribonacci substitution seems linked to an automorphism of surface. However, as several people noticed, there seems to be a contradiction here: every diffeomorphism of surface with orientable invariant foliation (this is the case here) has an homology matrix with a reciprocal characteristic polynomial, since it preserves the intersection form; but this is not the case for Tribonacci substitution. This was a starting point for this research, and the answer is that the Tribonacci partition is not generating; the finer partition we found gives a substitution which factors on Tribonacci substitution, and whose polynomial is generating.

In this particular case, it happens that we get the same fractal sets for expanding and contracting directions; it is unclear whether this holds true for all reciprocal substitutions, or at least for all substitutions associated with self-induced interval exchanges.

The sets $K_{i}$ can be interpreted easily in the symbolic system, and also on the flat surface, as 3 segments of separatrices near a singular point. But they also appear, by projection on the stable space $E_{T, s}$, as a partition of the Tribonacci fractal, and the corresponding sets seem to tile the plane; is it really the case, and what is the meaning of the corresponding lattice?

8.2. Duality and higher dimensional objects. In the present example, we were able to recover a dual substitution, because we knew the underlying interval exchange. It remains to find a general theory which allows, when possible, to build a dual substitution. A dual substitution, which acts on faces of codimension 1, has been defined in [5]; but its relation with the substitution built here is not clear.

A general notion of higher dimensional extensions of substitutions and their dual maps has been defined in [6], and used in [3], [4]; it would seem natural to use it in this framework, to approximate the stable and unstable space; the fact that the present substitution is reducible, and the existence of a nontrivial neutral space, would probably make things more difficult for the present example. Note that some progress has been made recently on group automorphisms and associated tilings.

There is a symbolic dynamical system naturally associated with a substitution; in the basic examples (Pisot irreducible substitutions), this symbolic dynamical system has a symbolic representation as an exchange of pieces, which turns out in all known cases to be a translation on a torus. In this paper, we have given another representation as an interval exchange (which is measurably conjugate to a rotation on a torus); the good notion to consider in more complicated cases (Reducible, or non-Pisot) is unclear; in some non-Pisot cases, it seems to be a pseudo-group of translations.

Remark 54. We already observed that the underlying GIFS had to be the same (double duality) up to translation vectors. These translation vectors depend on the prefixes (and hence on the order) and on the basis.

8.3. Bi-infinite sequences. For the sake of simplicity, we have mainly used infinite sequences, but of course it might be more natural, if somewhat cumbersome, to use biinfinite sequences. In that case, there are countably many infinite sequences which can be extended in several different ways on the left, and only a finite number of orbits which branch at some point, with sequences which have the same past and different futures (or the converse). 
In the present case, these special sequences can be interpreted as the discontinuity points of the interval exchange, where the image of a point can be defined differently using continuity on the right and continuity on the left.

It would also be interesting to study the bi-infinite fixed points on the dual substitution; there seems to be 12 of them, twice more than for the initial substitution.

8.4. A more symmetric version. Increasing the number of letters, we can find another partition and corresponding to another substitution

$$
\begin{aligned}
\sigma_{s}: 1_{A} & \rightarrow 2_{B} 3_{A} 3_{B} 4_{A} \\
1_{B} & \rightarrow 4_{B} 5_{A} 5_{B} \\
2_{A} & \rightarrow 6_{A} 6_{B} 1_{A} \\
2_{B} & \rightarrow 1_{B} 2_{A} \\
3_{A} & \rightarrow 1_{A} \\
3_{B} & \rightarrow 1_{B} \\
4_{A} & \rightarrow 2_{A} \\
4_{B} & \rightarrow 2_{B} \\
5_{A} & \rightarrow 3_{A} \\
5_{B} & \rightarrow 3_{B} \\
6_{A} & \rightarrow 4_{A} \\
6_{B} & \rightarrow 4_{B} .
\end{aligned}
$$

Of course, the Abelianization map has a three dimensional null space showing that nothing new happens, but this recoding may be useful in that it stresses better the symmetries. The correspondence is :

$$
1 \approx 1_{A}, 2 \approx 1_{B}, 3 \approx 2_{A}, 4 \approx 2_{B}, 5 \approx 3_{A} 3_{B}, 6 \approx 4_{A}, 7 \approx 4_{B}, 8 \approx 5_{A} 5_{B}, 9 \approx 6_{A} 6_{B} .
$$

\section{APPENDiX}

9.1. Abelianization. The matrix of the substitution $\sigma$ in the canonical basis is

$$
M=M_{\sigma}=\left(\begin{array}{lllllllll}
0 & 0 & 0 & 1 & 1 & 1 & 0 & 0 & 0 \\
0 & 0 & 0 & 0 & 0 & 0 & 1 & 1 & 0 \\
1 & 0 & 0 & 0 & 0 & 0 & 0 & 0 & 1 \\
0 & 1 & 1 & 0 & 0 & 0 & 0 & 0 & 0 \\
1 & 1 & 0 & 0 & 0 & 0 & 0 & 0 & 0 \\
0 & 0 & 1 & 0 & 0 & 0 & 0 & 0 & 0 \\
0 & 0 & 0 & 1 & 0 & 0 & 0 & 0 & 0 \\
0 & 0 & 0 & 0 & 1 & 0 & 0 & 0 & 0 \\
0 & 0 & 0 & 0 & 0 & 1 & 1 & 0 & 0
\end{array}\right) .
$$

We choose a new basis $\widetilde{\mathcal{B}}=\left\{\tilde{e}_{i}, i=1, \ldots, 9\right\}$ adapted to decomposition $\mathbb{R}^{\mathcal{A}}=E_{n} \oplus E_{T} \oplus$ $E_{\bar{T}}$. Since these subspaces are given by rational equations, it is possible to choose vectors with integral coordinates (while it is not possible for the finer decomposition in stable and ustable spaces, because this finer decomposition is not given by rational equations); there are many possible choices. For convenience in computations, we will use half integers. We denote $P$ the change of basis ; its column vectors are the coordinates, in the canonical 
basis, of the vectors of the new basis.

$$
P=\frac{1}{2}\left(\begin{array}{rrrrrrrrr}
0 & 0 & 0 & 0 & 2 & 0 & 1 & 0 & -1 \\
2 & 0 & 0 & -1 & -1 & 1 & 0 & 0 & 1 \\
-2 & 0 & 0 & 1 & -1 & 1 & 0 & 1 & 0 \\
0 & 0 & 0 & 0 & 0 & -2 & 1 & -1 & 0 \\
0 & 2 & 0 & 1 & -1 & 1 & 0 & 1 & 0 \\
0 & -2 & 0 & 1 & 1 & -1 & 0 & 0 & 1 \\
0 & 0 & 0 & -2 & 0 & 0 & 0 & 1 & -1 \\
0 & 0 & 2 & 1 & 1 & -1 & 0 & 0 & 1 \\
0 & 0 & -2 & -1 & -1 & 1 & 0 & 0 & 1
\end{array}\right) .
$$

If $J=\left(\begin{array}{lll}0 & 0 & 1 \\ 1 & 0 & 0 \\ 0 & 1 & 0\end{array}\right)$ denotes the permutation matrix, and $A=\left(\begin{array}{lll}1 & 1 & 1 \\ 1 & 0 & 0 \\ 0 & 1 & 0\end{array}\right)$ the Tribonacci matrix, then the matrix $\widetilde{M}$ corresponding to $M$ in the new basis writes

$$
\widetilde{M}=P^{-1} M P=\left(\begin{array}{rrr}
J & 0 & 0 \\
0 & { }^{t} A^{-1} & 0 \\
0 & 0 & A
\end{array}\right)
$$

9.2. Arithmetic basis. To give an arithmetical interpretation of the projections of the stepped line on the subspaces $E_{n}$ and $E_{\bar{T}}$, we consider another basis $\hat{\mathcal{B}}$ of these subspaces which allows us to recover the arithmetical properties of the IET.

Namely, we look for a projection on a suitable subspace, and a basis of this subspace, such that the coordinates of the projections of the vectors of the canonical basis in this basis correspond to the coordinates in the natural basis of $\mathbb{Z}[\alpha]$ of the corresponding translations for the interval exchange map. It is in fact convenient to define this basis on the complete space $\mathbb{R}^{\mathcal{A}}$.

We define the basis $\hat{\mathcal{B}}$ by its coordinates in the canonical basis by the matrix :

$$
\hat{P}=\frac{1}{12}\left(\begin{array}{rrrrrrrrr}
0 & 0 & 0 & -6 & 0 & -6 & 6 & 0 & -6 \\
6 & 10 & -2 & 3 & 3 & 3 & 0 & 0 & 6 \\
-6 & -10 & 2 & -3 & -3 & 3 & 0 & 6 & 0 \\
0 & 0 & 0 & 6 & 0 & 0 & 6 & -6 & 0 \\
6 & 4 & 4 & -3 & -3 & 3 & 0 & 6 & 0 \\
-6 & -4 & -4 & -3 & -3 & -3 & 0 & 0 & 6 \\
0 & 0 & 0 & 6 & 6 & 0 & 0 & 6 & -6 \\
6 & 4 & -2 & -3 & -3 & -3 & 0 & 0 & 6 \\
-6 & -4 & 2 & 3 & 3 & 3 & 0 & 0 & 6
\end{array}\right) \text {. }
$$

The first vector $\hat{e}_{1}$ is the invariant vector, the first three vectors $\hat{e}_{1}, \hat{e}_{2}, \hat{e}_{3}$ form a basis $\hat{\mathcal{B}_{N}}$ of $E_{n}$; the next three vectors $\hat{e}_{4}, \hat{e}_{5}, \hat{e}_{6}$ form a basis $\hat{\mathcal{B}_{\bar{T}}}$ of $E_{\bar{T}}$, and the last three form a basis of $E_{T}$ (these last three vectors are not changed from the basis $\widetilde{\mathcal{B}}$, and will play no role in what follows).

Definition 55. We denote by $F$ the subspace generated by $\hat{e}_{1}-\hat{e}_{4}, \hat{e}_{2}-\hat{e}_{5}, \hat{e}_{3}-\hat{e}_{6}, \hat{e}_{7}, \hat{e}_{8}, \hat{e}_{9}$.

We denote by $\Pi_{F}$ the projection on $E_{\bar{T}}$ along $F$.

We denote $\Pi_{E_{j} \oplus E_{\bar{T}}}$ the projection on the subspace $E_{j} \oplus E_{\bar{T}}$ along the subspace $E_{I} \oplus E_{T}$. We define the map $\hat{\Pi}=\Pi_{F} \circ \Pi_{E_{j} \oplus E_{\bar{T}}}$. 
The map $\hat{\Pi}$ is a projection; direct computation shows that it is a projection on $E_{\bar{T}}$ along the space generated by $\left\{\hat{e}_{1}, \hat{e}_{2}+\hat{e}_{4}-\hat{e}_{5}, \hat{e}_{3}-\hat{e}_{6}, \hat{e}_{7}, \hat{e}_{8}, \hat{e}_{9}\right\}$, and allows us to recover its matrix:

Lemma 56. If we consider the projection $\hat{\Pi}$ as a map from $\mathbb{R}^{\mathcal{A}}$, with the canonical basis $\mathcal{B}$, to $E_{\bar{T}}$, with the basis $\left\{\hat{e}_{4}, \hat{e}_{5}, \hat{e}_{6}\right\}$, its matrix is:

$$
\left(\begin{array}{rrrrrrrrr}
-1 & -1 & 1 & 1 & -1 & -1 & 1 & 2 & 0 \\
1 & 1 & -1 & -1 & 0 & 0 & 0 & -1 & 1 \\
0 & 0 & 0 & 0 & 1 & -1 & -1 & -1 & 1
\end{array}\right)
$$

Recall that, for a 3-dimensional space $E$ with a basis $\mathcal{B}$, we denote by $Q_{E, \mathcal{B}}$ the map defined on the lattice generated by $\mathcal{B}$ by $Q_{E, \mathcal{B}}(v)=\frac{n}{2}+\frac{p}{2} \alpha+\frac{q}{2} \alpha^{2}$, where $(n, p, q)$ are the coordinates of $v$ in the basis $\mathcal{B}$.

The essential result of this section is:

Lemma 57. The projections on $E_{\bar{T}}$ of the canonical vectors satisfy

$$
\left.Q_{E_{\bar{T}}, \hat{\mathcal{B}}}\left(\hat{\Pi}\left(e_{i}\right)\right)\right)=t_{i}
$$

Proof. This is a direct application of the previous lemma; we observe that applying $Q$, to the matrix above, we obtain exactly the displacement vector

$$
\frac{1}{2}\left(\alpha-1, \alpha-1,1-\alpha, 1-\alpha, \alpha^{2}-1,-1-\alpha^{2}, 1-\alpha^{2}, 2-\alpha-\alpha^{2}, \alpha+\alpha^{2}\right) .
$$

Remark 58. This lemma makes all further computations straightforward; it makes clear the link between what happens in the symbolic space and the more arithmetic description of the system.

There is a simple description of the projection $\hat{\Pi}$. Remark that the projection $\Pi_{F}$ is the identity on $E_{\bar{T}}$, the zero map on $E_{T}$, and sends $E_{n}$ to $E_{\bar{T}}$ by a map which sends $\hat{e}_{i}$ to $\hat{e}_{i+3}$, for $i=1,2,3$.

We can describe $\tilde{\Pi}$ simply by abusing the language: let us identify $E_{n}$ ( with basis $\left\{\hat{e}_{1}, \hat{e}_{2}, \hat{e}_{3}\right\}$ ) and $E_{\bar{T}}$ (with basis $\left\{\hat{e}_{4}, \hat{e}_{5}, \hat{e}_{6}\right\}$ ) to $\mathbb{R}^{3}$ with the canonical basis, so that it makes sense to add vectors in these two different spaces. Consider $\Pi_{j}$ as a map from $\mathbb{R}^{\mathcal{A}}$ to $\mathbb{R}^{3}$, and $\Pi_{\bar{T}}$ also as a map from $\mathbb{R}^{\mathcal{A}}$ to $\mathbb{R}^{3}$.

The very definition of $\hat{\Pi}$ shows that we have, with this abuse of language:

$$
\hat{\Pi}(V)=\Pi_{\bar{T}}(V)+\Pi_{j}(V) .
$$

Since the projection $\Pi_{j}$ takes only 3 values on the canonical stepped line, as we saw above, the difference between $\tilde{\Pi}$ and $\Pi_{\bar{T}}$ on the canonical line also can take only three values; McMullen's curve differs form the standard projection of the canonical line on $E_{\bar{T}, u}$ by a finite set of translations. 
9.3. Proof of Lemma 53. Let us do the complete computation to show $A w_{P_{0}^{*}}+w_{P_{a_{0}}}-$ $A w_{P_{a_{1}}^{W}}=e_{\rho\left(P_{0}\right)}+e_{\rho\left(a_{0}\right)}-A e_{\rho\left(a_{1}\right)}$. We recall that $w_{a}$ stands for $\Pi \Pi_{\bar{T}}^{*}\left(e_{i}\right)$. We observe that $E_{\bar{T}}^{*}$ is indeed determined by the system of linear equations

$$
\left\{\begin{array}{r}
w_{1}+w_{2}+w_{3}+w_{4}=0 \\
w_{5}+w_{6}+w_{7}=0 \\
w_{8}+w_{9}=0 \\
w_{2}+w_{6}=0 \\
w_{6}-w_{8}=0 \\
w_{3}-w_{5}=0
\end{array} .\right.
$$

It is hence clear that $e_{a}=-w_{7}, e_{b}=w_{1}$ and $e_{c}=-w_{4}$ is a basis. We recall that in this basis, the restriction of ${ }^{t} M^{-1}$ acts as the Tribonacci matrix $A$. The other vectors are

$$
\left\{\begin{array}{l}
w_{2}=\frac{1}{2}\left(-e_{a}-e_{b}+e_{c}\right) \\
w_{3}=\frac{1}{2}\left(e_{a}-e_{b}+e_{c}\right) \\
w_{5}=\frac{1}{2}\left(e_{a}-e_{b}+e_{c}\right) \\
w_{6}=\frac{1}{2}\left(e_{a}+e_{b}-e_{c}\right) \\
w_{8}=\frac{1}{2}\left(e_{a}+e_{b}-e_{c}\right) \\
w_{9}=\frac{1}{2}\left(-e_{a}-e_{b}+e_{c}\right)
\end{array} .\right.
$$

The 16 cases corresponding to the 16 edges of the prefix automaton are listed below.

\begin{tabular}{llllllllll}
$a_{0} \rightarrow a_{1}$ & $\rho\left(a_{0}\right) \stackrel{\rho\left(P_{0}\right)}{\rightarrow} \rho\left(a_{1}\right)$ & $\sigma_{*}\left(P_{0}^{W}\right) P_{0}^{*}$ & $w_{a_{0}}+$ & $\left.A w_{P_{0}^{*}}-A\left(w_{a_{1}}\right)\right)=e_{\rho\left(P_{0}\right)}+e_{\rho\left(a_{0}\right)}-A e_{\rho\left(a_{1}\right)}=$ \\
\hline $3 \rightarrow 9$ & $a \stackrel{\epsilon}{\rightarrow} c$ & $\sigma_{*}(\epsilon) \epsilon=\epsilon$ & 0 & 0 & 0 & 0 & $e_{a}$ & $A e_{c}$ & 0 \\
$3 \rightarrow 1$ & $a \stackrel{\epsilon}{\rightarrow} a$ & $\sigma_{*}(\epsilon) 9$ & 0 & $A w_{9}$ & $A w_{34}$ & 0 & $e_{a}$ & $A e_{a}$ & $-e_{b}$ \\
$4 \rightarrow 2$ & $a \stackrel{\epsilon}{\rightarrow} a$ & $\sigma_{*}(3) \epsilon$ & $w_{3}$ & $A 0$ & $A w_{341}$ & 0 & $e_{a}$ & $A e_{a}$ & $-e_{b}$ \\
$4 \rightarrow 3$ & $a \stackrel{\epsilon}{\rightarrow} a$ & $\sigma_{*}(3) 2$ & $w_{3}$ & $A w_{2}$ & 0 & 0 & $e_{a}$ & $A e_{a}$ & $-e_{b}$ \\
$1 \rightarrow 4$ & $a \stackrel{\epsilon}{\rightarrow} a$ & $\sigma_{*}(34) \epsilon$ & $w_{34}$ & 0 & $A w_{3}$ & 0 & $e_{a}$ & $A e_{a}$ & $-e_{b}$ \\
$1 \rightarrow 5$ & $a \stackrel{\epsilon}{\rightarrow} b$ & $\sigma_{*}(34) 4$ & $w_{34}$ & $A w_{4}$ & $A w_{67}$ & 0 & $e_{a}$ & $A e_{b}$ & $-e_{c}$ \\
$1 \rightarrow 6$ & $a \stackrel{\epsilon}{\rightarrow} b$ & $\sigma_{*}(34) 45$ & $w_{34}$ & $A w_{45}$ & 0 & 0 & $e_{a}$ & $A e_{b}$ & $-e_{c}$ \\
$2 \rightarrow 7$ & $a \stackrel{\epsilon}{\rightarrow} b$ & $\sigma_{*}(341) \epsilon$ & $w_{341}$ & 0 & $A w_{6}$ & 0 & $e_{a}$ & $A e_{b}$ & $-e_{c}$ \\
$2 \rightarrow 8$ & $a \stackrel{\epsilon}{\rightarrow} c$ & $\sigma_{*}(341) 7$ & $w_{341}$ & $A w_{7}$ & $A w_{9}$ & 0 & $e_{a}$ & $A e_{c}$ & 0 \\
$6 \rightarrow 3$ & $b \stackrel{a}{\rightarrow} a$ & $\sigma_{*}(3412) \epsilon$ & 0 & 0 & 0 & $e_{a}$ & $e_{b}$ & $A e_{a}$ & 0 \\
$7 \rightarrow 4$ & $b \stackrel{a}{\rightarrow} a$ & $\sigma_{*}(34126) \epsilon$ & $w_{6}$ & 0 & $A w_{3}$ & $e_{a}$ & $e_{b}$ & $A e_{a}$ & 0 \\
$5 \rightarrow 1$ & $b \stackrel{a}{\rightarrow} a$ & $\sigma_{*}(341267) \epsilon$ & $w_{67}$ & 0 & $A w_{34}$ & $e_{a}$ & $e_{b}$ & $A e_{a}$ & 0 \\
$5 \rightarrow 2$ & $b \stackrel{a}{\rightarrow} a$ & $\sigma_{*}(341267) 1$ & $w_{67}$ & $A w_{1}$ & $A w_{341}$ & $e_{a}$ & $e_{b}$ & $A e_{a}$ & 0 \\
$9 \rightarrow 6$ & $c \stackrel{a}{\rightarrow} b$ & $\sigma_{*}(3412675) \epsilon$ & 0 & 0 & 0 & $e_{a}$ & $e_{c}$ & $A e_{b}$ & 0 \\
$9 \rightarrow 7$ & $c \stackrel{a}{\rightarrow} b$ & $\sigma_{*}(3412675) 6$ & 0 & $A w_{6}$ & $A w_{6}$ & $e_{a}$ & $e_{c}$ & $A e_{b}$ & 0 \\
$8 \rightarrow 5$ & $c \stackrel{a}{\rightarrow} b$ & $\sigma_{*}(34126759) \epsilon$ & $w_{9}$ & 0 & $A w_{67}$ & $e_{a}$ & $e_{c}$ & $A e_{b}$ & 0
\end{tabular}

\section{REFERENCES}

[1] Arnoux, P. Un exemple de semi-conjugaison entre un échange d'intervalles et une translation sur le tore. (French) [An example of semiconjugacy between an interval exchange and a shift on the torus] , Bull. Soc. Math. France 116 (1988), no. 4, 489-500.

[2] Arnoux, P ; Yoccoz, J.-C. Construction de difféomorphismes pseudo-Anosov, C. R. Acad. Sci. Paris, Sér. I Math. 292 (1981), no. 1, 75-78.

[3] P. Arnoux, V. Berthé, S. Ito, Discrete planes, $\mathbb{Z}^{2}$-actions, Jacobi-Perron algorithm and substitutions, Ann. Inst. Fourier (Grenoble) 52 (2002), 1001-1045. 
[4] P. Arnoux, V. Berthé, A. Siegel, Two-dimensional iterated morphisms and discrete planes, Theoret. Comput. Sci. 319 (2004), 145-176.

[5] P. Arnoux, S. Ito, Pisot substitutions and Rauzy fractals, Bull. Belg. Math. Soc. Simon Stevin 8 (2001), 181-207.

[6] Arnoux, P ; Ito, S ; Sano, Y, Higher dimensional extensions of substitutions and their dual maps, Journal d'Analyse Mathématique 83 (2001), 183-206.

[7] Bowman, J. Orientation-reversing involutions of the genus 3 Arnoux-Yoccoz surface and related surfaces, Preprint arXiv:0812.3144v1

[8] Dumont, J.-M. ; Thomas, A. Digital sum moments and substitutions, Acta Arith. 64 (1993), no. 3, 205-225.

[9] Fathi, A. Some compact invariant sets for hyperbolic linear automorphisms of tori, Ergod. Th. \& Dynam. Sys. 8 (1988), 191-204.

[10] Fogg, N. Pytheas, Substitutions in dynamics, arithmetics and combinatorics., Edited by V. Berthé, S. Ferenczi, C. Mauduit and A. Siegel. Lecture Notes in Mathematics, 1794. Springer-Verlag, Berlin, 2002.

[11] Hubert, P. ; Lanneau, E. Veech groups without parabolic elements, Duke Math. J. 133 (2006), 335346.

[12] Hubert, P. ; Lanneau, E. ; Möller, M. The Arnoux-Yoccoz Teichmüller disc, Geom. Funct. Anal. 18 (2009) no. 6, 1988-2016.

[13] Lowenstein, J. H. ; Poggiaspalla, G. ; Vivaldi, F. Interval exchange transformations over algebraic number fields: the cubic Arnoux-Yoccoz model, Dyn. Syst. 22 (2007), no. 1, 73-106.

[14] McMullen, C.T. http://abel.math.harvard.edu/ ctm/gallery/billiards/ arnoux.gif

[15] Messaoudi, A. Frontière du fractal de Rauzy et système de numération complexe, Acta Arith. 95 (2000), no. 3, 195-224.

[16] Nipper, E Minimlality and nonergodicity on a family of flat surfaces in genus 3, Preprint arXiv:0801.0466v1

[17] Rauzy, G. Nombres algébriques et substitutions Bull. S.M.F. 110 (1982), 147-178.

[18] Sirvent, V. Identifications and dimension of the Rauzy fractal. Fractals 5 (1997), no. 2, 281-294.

[19] Sirvent, V. Geodesic laminations as geometric realizations of Pisot substitutions. Ergodic Theory Dynam. Systems 20 (2000), no. 4, 1253-1266.

(Arnoux) Institut de Mathématiques de Luminy, CNRS U.M.R. 6206, 163, AVenue De LuMiny, Case 907, 13288 Marseille Cedex 09 France

E-mail address, Arnoux: arnoux@iml.univ-mrs.fr

(Bernat) IUfM de Lorraine et Institut Elie Cartan, CNRS U.M.R. 7502, Université Henri Poincaré Nancy 1 B.P. 239, F-54506 Vandoeuvre-Lés-Nancy Cedex

E-mail address, Bernat: julien.bernat@lorraine.iufm.fr

(Bressaud) Université Paul Sabatier, Institut de Mathématiques de Toulouse, F-31062 Toulouse Cedex 9, France

E-mail address, Bressaud: bressaud@ math.univ-toulouse.fr 